\title{
Exploring the Relationship between Gut Microbiota and Major Depressive Disorders
}

\author{
Catherine $\operatorname{Tian}^{1}$ \\ ${ }^{1}$ Shanghai American School, Shanghai, China
}

\begin{abstract}
Major Depressive Disorder (MDD) is a psychiatric disorder accompanied with a high rate of suicide, morbidity and mortality. With the symptom of an increasing or decreasing appetite, there is a possibility that MDD may have certain connections with gut microbiota, the colonies of microbes which reside in the human digestive system. In recent years, more and more studies started to demonstrate the links between MDD and gut microbiota from animal disease models and human metabolism studies. However, this relationship is still largely understudied, but it is very innovative since functional dissection of this relationship would furnish a new train of thought for more effective treatment of MDD. In this study, by using multiple genetic analytic tools including Allen Brain Atlas, genetic function analytical tools, and MicrobiomeAnalyst, I explored the genes that shows both expression in the brain and the digestive system to affirm that there is a connection between gut microbiota and the MDD. My approach finally identified 7 MDD genes likely to be associated with gut microbiota, implicating 3 molecular pathways: (1) Wnt Signaling, (2) citric acid cycle in the aerobic respiration, and (3) extracellular exosome signaling. These findings may shed light on new directions to understand the mechanism of MDD, potentially facilitating the development of probiotics for better psychiatric disorder treatment.
\end{abstract}

\section{Introduction}

\subsection{Major Depressive Disorder}

Major Depressive Disorder (MDD) is a mood disorder that will affect the mood, behavior and other physical parts. MDD will be accompanied by depression, decreased interest and fun in activities, weight loss and decreased or increased appetite, frequent insomnia, psychomotor agitation or mental retardation, suicidal awareness but no specific plan, and so forth [1]. The prevalence of depressive disorder occurs all over the world (Figure 1,2), and there is no definite medicine or treatments that can completely cure depressive disorder. In 2017, the number given by the WHO shows that there are 17.3 million adults had at least one Major Depressive episode, which is $7.1 \%$ of the US adult [2]. Some of the risk factors for MDD in previous studies show that females have a higher prevalence and risk of MDD [3]. Also, genetic influence and age is also a risk factor of the morbidity of MDD. Study shows that the onset of MDD is low until the early adolescence [4].

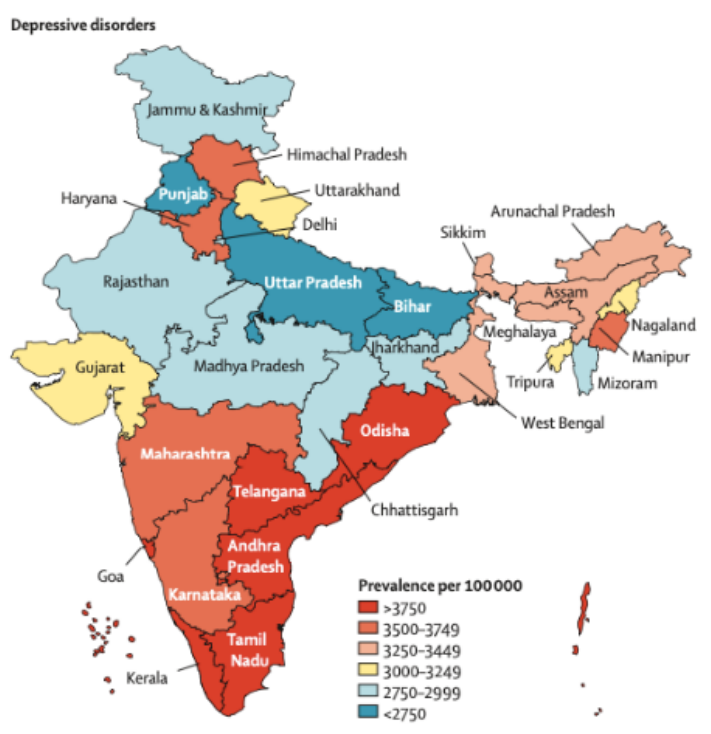

Fig. 1. Crude prevalence of depressive disorders in India, 2017 [5]. 


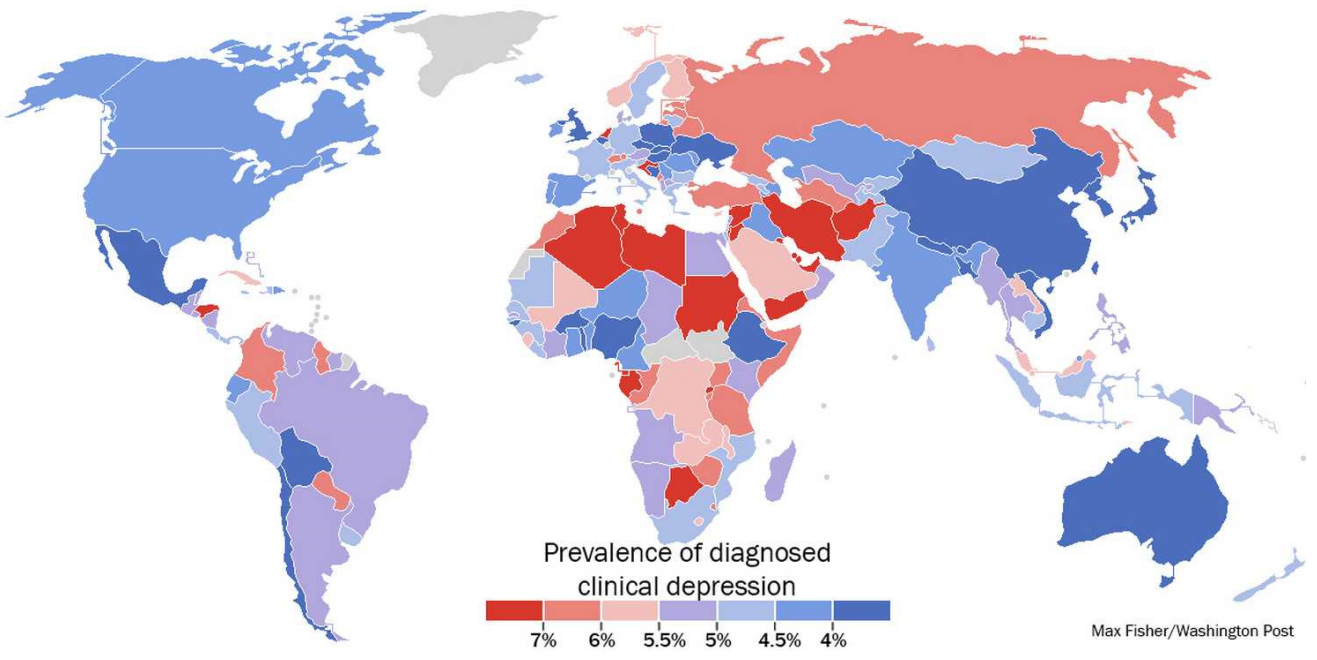

Fig. 2. Worldwide crude prevalence of depressive disorders, 2013 [6].

\subsection{Major Depressive Disorder}

Currently available treatments for MDD include antidepressant medications such as selective serotonin reuptake inhibitors (SSRIs), serotonin and norepinephrine reuptake inhibitors (SNRIs), tricyclic antidepressants (TCAs) and psychological therapy [7]. However, these antidepressant medicines also have some severe side effects such as for SSRI, it will reduce the concentration of the neurotransmitter serotonin in platelets, it will lead to a decrease in blood clotting ability [8]. Besides, when someone had experienced severely lost such as death or job loss, the antidepressant drugs may not work [9]. Another disorder that can also produce distressing psychotic symptoms like MDD is bipolar disorders; these two disorders can lead to highly disabling and unpleasant depressive episodes [10].

The key to finding more effective treatments for MDD and bipolar disorder is the exploration of their molecular mechanisms. Previous genetic studies have identified several genes whose mutation can lead to MDD. For example, glucocorticoid receptor gene $\mathrm{NR} 3 \mathrm{C} 1$ and glycogen synthase kinase $3 \beta$ is associated with a higher risk of MDD, implicating its potential connection with hormone regulation and metabolism [11]. In other published studies, it has been shown that the patients who suffer in MDD has a decreased serum level of BDNF [12]. Although BDNF plays multiple roles in the function of central nervous system, its detailed molecular mechanisms of action on MDD have not been further explored.

\subsection{Gut Microbiota and its Potential Connection to MDD}

It has long been appreciated that diet and food intake are associated with the onset of Major Depressive

Depression. For instance, individuals who are lack many nutrients such as B-complex vitamins and other acids such as Lithium often develop mental illness [13]. Recent psychiatric studies have shown that some of the MDD symptoms include increasing or decreasing appetite [14]. Thus, I hypothesize that MDD might be linked to the enteric microorganisms, or the so-called "gut microbiota", which is a component that has already been shown to associated with many brain disorders.

The gut microbiota contains over 3 million genes [15] and 100 trillion microorganisms which can influence our well-being in many significant aspects [16]. Recent studies have suggested a stunning connection between Parkinson's Disease (PD) and gut microbiota (such as Proteus sp. and Bilophila sp.) [17]. The so-called "braingut axis" is reported to directly contribute to the aggregation of PD causing $\alpha$-synuclein proteins in the midbrain, by dysfunction in microglia activation. Progressively preclinical literature indicates that there is bidirectional signal transduction between the brain and gut microbiome, which involves a variety of neuroendocrine and endocrine signal transduction mechanisms [18]. Research shows that probiotic administration not only affects the fMRI in the brain but also causes a change in the urine metabolic profile, further proves the hypothesis that there is bidirectional signal transduction between the brain and the gut microbiome [19].

Another important aspect to link MDD and gut microbiota is the immune system. In the past few decades, several published studies have already demonstrated that MDD is usually associated with changes in the immune system. For instance, MDD patients are often characterized by elevated levels of proinflammatory cytokines in the circulation and activation of microglia in the brain [20]. On the other hand, the gut microbiota is a part of the immune system and it is also one of the symptoms of MDD [21]. Therefore, I speculate that gut microbiota may be related to depression.

\subsection{Rationale and Aim}

Based on the above rationale, I aim to learn about the relationship between MDD and gut microbiota. The relationship could be a very interesting exploration strategy and may shed new insight on both the molecular mechanisms of MDD and aid on better treatments of 
MDD. Based on multiple datasets including genomic, metabolic, and microbiological information, I first conducted a computational screen to filtrate these genes that might have a relationship between MDD and the gut microbiota. Then, I further present a gene network analysis to further analyze the relationship between these genes that has been filtrated already. Lastly, I carried out a systematic microbiome analysis on the candidate MDD gene list to explore their relationship with gut microbiota.

\section{Materials and Methods}

\subsection{Data Sources}

Several publicly accessible databases of neurobiology and microbiology were employed in this paper. They are summarized in the following sections.

\subsubsection{Allen Brain Atlas}

Allen Brain Atlas is a comprehensive and multidisciplinary database combing the genomic, transcriptomic, neuroanatomy, and connectome data of the mouse and human brains [22]. It contains 16 databases about the mouse and human cell types database and brain atlas. Allen Brain Atlas also provides data visualizing tools such as Brain Explorer and Planner View for researchers to grasp a comprehensive understanding of the nervous under physiological and disease circumstances.

The Allen Brain Atlas database is accessible from the following link: https://portal.brain-map.org/

\subsubsection{NIH Human Microbiome Project}

1) Human Microbiota Project (HMP) \& Integrative Human Microbiota Project (iHMP)

HMP and iHMP were created under the project NIH Human Microbiome Project. iHMP is the second phase of HMP. HMP generates resources to promote the characterization of the human microbiome to further understand how the microbiome affects human health and disease. iHMP is engaging in providing new calculation tools and comprehensive molecular perspectives on microbial activity during metabolic abnormalities. The database HMP and iHMP contain 31,596 samples, and a total of 161,256 files provided to all researchers. It is characterized by a microbial community from 300 healthy individuals spread across several different parts of the human body: nasal passages, mouth, skin, gastrointestinal tract, and urogenital tract. HMP contains the following parts:

The HMP database is accessible from the following link: https://www.hmpdacc.org/hmp/

The iHMP database is accessible from the following link: https://www.hmpdacc.org/ihmp/

\subsubsection{GeneCards}

GeneCards is a database that provides human genes that were predicted and annotated. The knowledge base contains more than 190 datasets to provide sources or different genes and parts that can be influenced by that gene. It provides a different section in the genes such as aliases, disorders, domains, drug, expression, function, genomics, localization, pathway, product, protein, publication, and many others in order to provide more detail and comprehensive understanding to researchers.

The GeneCards database is accessible from the following link: https://www.genecards.org/

\subsubsection{Computational Tools and Algorithms}

\section{1) STRING}

STRING is a well-known online genetic analytic web resource and database, which can predict protein-protein interaction and depict the interaction network. Experimental data, computational prediction methods and public text collections are all collected in the STRING database.

The STRING database is accessible from the following link: https://string-db.org/

2) Database for Annotation, Visualization and Integrated Discovery (DAVID)

DAVID is a comprehensive biological knowledge base and analysis tool designed to systematically extract biological meaning from large gene lists [23]. Using high-throughput genomics, proteomics, and bioinformatics scanning methods to perform functional analysis of large gene lists is still a difficult task [24]. DAVID allows researchers to gain insight into the biological topics in the gene list in genome-scale research [23].

DAVID is accessible from the following link: https://david.ncifcrf.gov/tools.jsp

\section{3) R programming language and R Studio}

$\mathrm{R}$ is a language and environment for statistical calculations and graphics and provides various statistical information and graphics techniques. $\mathrm{R}$ can easily produce well-designed publication-quality charts, including the required mathematical symbols and formulas. When using R, g: Profiler is also involved. g: Profiler tool set can be used to find different biological categories in the gene list, conversion between gene identifiers, and so on [25].

4) MicrobiomeAnalyst

MicrobiomeAnalyst contains 4 sections in visual and meta-analysis of microbiome data, it includes: Marker Data Profiling (MDP), Shoutgun Data Profiling (SDP), Projection with Public Data (PPD), Taxon Set Enrichment Analysis (TSEA) [26].

The MicrobiomeAnalst database is accessible from the following link: https://www.microbiomeanalyst.ca/ MicrobiomeAnalyst/home.xhtml 


\subsubsection{Project Workflow}

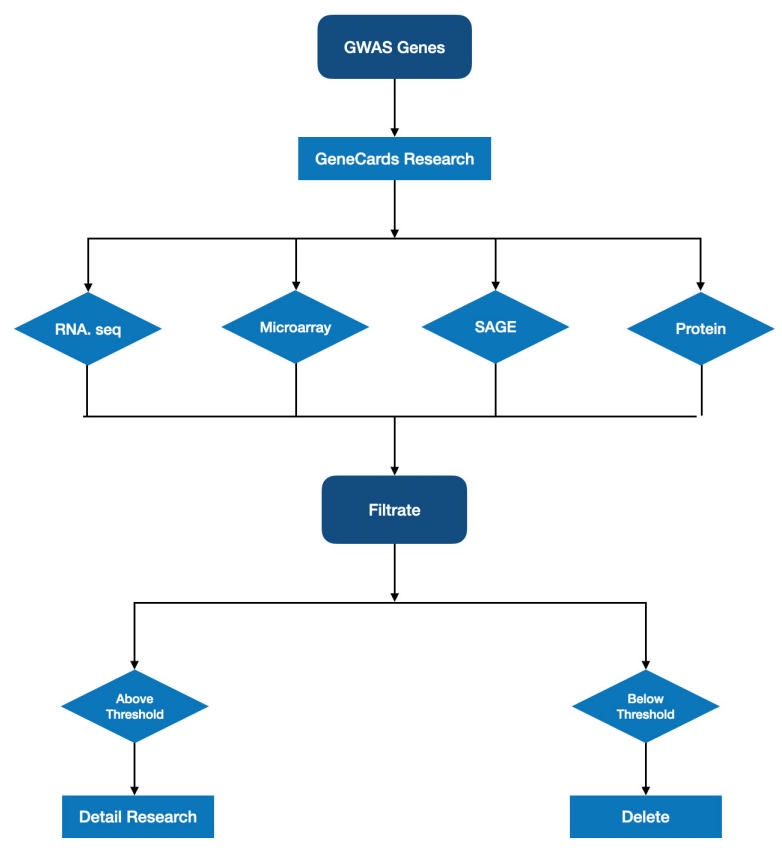

Fig. 3. Screening Process in GWAS genes.

This is a flowchart showing the screening process for GWAS genes. At first, I filtrate all the non-proteincoding genes, then I rate them through Genecards data. Last, I set a threshold for the genes, if the scores are above the threshold, I will have detailed research on that gene to see if it really has a relationship between the brain and gut microbiota.

\section{Results}

\subsection{Computational screen for potential gut microbiota related MDD genes}

The key question to reveal the relationship between MDD and gut microbiota is to ensure there are genes that are both related to MDD and gut microbiota. In order to address these questions, I decided to conduct a computational screen of MDD related genes that might be involved in the regulation of gut microbiota or the digestive system. Conducting a computational screen at the first step helps to integrate the information of the genes which helps to narrow down the genes that shows both high expression in MDD and gut microbiota. By using a computational screen, it helps me to filtrate genes and finally find out the genes that is related to MDD and gut microbiota.

First, I examined all relatively well-characterized MDD genes to confirm their RNA and protein expressions in the brain and to explore whether they may also be expressed in the digestive system. This will help us demonstrate if any of these genes might be involved in the brain-gut axis. Secondly, I employed a similar computational approach to search novel genetic candidates based on a more recent genome-wide association study (GWAS) that has identified 44 risk variants [27]. Lastly, I examine the molecular functions of these candidates through another computational screen.

\subsubsection{Conventional MDD related genes}

To start with, I perform a literature search on the Pubmed publication database, examining Systematic Reviews mentioning "gut microbiota" and "Major Depressive Disorder". The search results suggest that there are approximately 12 genes that have been well appreciated in the psychiatric research community [28]. To better visualize the categories and functions of these genes, I then summarize these genes in Table 1.

Table 1. Summary of well-known genes in MDD

\begin{tabular}{|c|c|c|}
\hline Gene Name & Category & Known Functions \\
\hline GRIK4 & $\begin{array}{l}\text { Glutamate-gated } \\
\text { ion channel }\end{array}$ & $\begin{array}{l}\text { By activating ligand-gated ion channels and G protein-coupled } \\
\text { membrane receptors, it will excite the neurotransmitter in the central } \\
\text { nervous system. }\end{array}$ \\
\hline BDNF & $\begin{array}{l}\text { Neurotrophic } \\
\text { factor }\end{array}$ & $\begin{array}{l}\text { Binding of this protein to its cognate receptor promotes neuronal } \\
\text { survival in the adult brain }\end{array}$ \\
\hline FKBP5 & $\begin{array}{l}\text { Chaperone and } \\
\text { stress response } \\
\text { protein }\end{array}$ & $\begin{array}{l}\text { Immunoregulation and basic cellular processes involving protein } \\
\text { folding and trafficking }\end{array}$ \\
\hline COMT & Methyltransferase & $\begin{array}{l}\text { Transfer of a methyl group from S-adenosylmethionine to } \\
\text { catecholamines }\end{array}$ \\
\hline GSK3及 & Kinase & Identified as phosphorylation and inactivator of glycogen synthase \\
\hline SLC6A4 & $\begin{array}{c}\text { Integral } \\
\text { membrane protein }\end{array}$ & $\begin{array}{l}\text { Transports the neurotransmitter serotonin from synaptic spaces into } \\
\text { presynaptic neurons }\end{array}$ \\
\hline TREK1 & $\begin{array}{l}\text { Lipid-gated ion } \\
\text { channel }\end{array}$ & $\begin{array}{l}\text { Negative regulator of glucose homeostasis and is involved in energy } \\
\text { metabolism, inflammation, ER-stress, mitochondrial dysfunction, and } \\
\text { apoptotic pathways }\end{array}$ \\
\hline CRHBP & $\begin{array}{l}\text { Glutamate-gated } \\
\text { ionic channel }\end{array}$ & The major excitatory neurotransmitter in the central nervous system \\
\hline IGF-1 & Growth factor & $\begin{array}{l}\text { A member of a family of proteins involved in mediating growth and } \\
\text { development }\end{array}$ \\
\hline
\end{tabular}




\begin{tabular}{ccl}
\hline IL1及 & Cytokine & $\begin{array}{l}\text { Involved in a variety of cellular activities, including cell proliferation, } \\
\text { differentiation, and apoptosis. }\end{array}$ \\
\hline IL6 & Cytokine & $\begin{array}{l}\text { Implicated in a wide variety of inflammation-associated disease } \\
\text { states, including susceptibility to diabetes mellitus and systemic } \\
\text { juvenile rheumatoid arthritis }\end{array}$ \\
\hline TNFa & $\begin{array}{c}\text { Inflammatory } \\
\text { factor }\end{array}$ & $\begin{array}{l}\text { Implicated in a variety of diseases, including autoimmune diseases, } \\
\text { insulin resistance, and cancer. }\end{array}$ \\
\hline
\end{tabular}

In order to better explore the genes whether might be associated with the brain-gut axis, I recorded each gene expression in the area of the brain and the organs of the digestive system. To reduce bias and better visualize the results, I introduced a gene expression metric system to analyze these data. In the RNA expression metrics, I subdivided the range of gene expression to four levels, which are: $0 \sim 1$ (score 1); 1 10 (score 2); 10 100 (score 3); 100 1000 (score 4). For the final RNA expression score by each method (RNA-seq, microarray or SAGE), it is the averaged expression score across all tissues considered (small intestine, colon, stomach, and esophagus). Then, for the protein expression metrics, there are 7 levels: $-2 \sim-1$ (score 1); $-1 \sim 0$ (score 2); 0 1 (score 3); 1 2 (score 4); 2 3 (score 5); 3 4 (score 6); 4 5 (score 7). Each tissue expression score adds up then divided by the total number of tissues (four tissue types considered: small intestine, colon, stomach, and esophagus). The symbol "/" represents that there is no expression data currently available in the GeneCard database (Table 2).

Table 2. RNA and protein expression levels in the human digestive system

\begin{tabular}{|c|c|c|c|c|c|}
\hline & \multicolumn{3}{|c|}{ RNA expression metrics } & \multirow{2}{*}{$\begin{array}{c}\text { Protein } \\
\text { expression score }\end{array}$} & \multirow{2}{*}{$\begin{array}{c}\text { Overall } \\
\text { Average score }\end{array}$} \\
\hline & $\begin{array}{c}\text { RNA-seq } \\
\text { score }\end{array}$ & Microarray score & SAGE score & & \\
\hline SLC6A4 & 2.25 & 2 & 0 & 1 & 1.38 \\
\hline GRIK4 & 2 & 2 & 1 & 1 & 1.5 \\
\hline BDNF & 2 & 2 & 0 & 1 & 1.25 \\
\hline FKBP5 & 3 & 2 & 3 & 3.67 & 2.92 \\
\hline COMT & 2 & 2 & 1 & 4 & 2.25 \\
\hline TREK1/KCNK2 & 3 & 2.5 & 1 & 1 & 1.88 \\
\hline GSK3B & 3 & 2 & 1 & 3.33 & 2.33 \\
\hline CRHBP & 2 & 2 & 1 & 1 & 1.5 \\
\hline IGF-1 & 3 & 2 & 0 & 1 & 1.5 \\
\hline IL-1 $\beta$ & 2.75 & 2 & 2 & 1 & 1.94 \\
\hline IL-6 & 3 & 2 & 1 & 1 & 1.75 \\
\hline TNF $\alpha$ & 2.25 & 2 & 1 & 1 & 1.56 \\
\hline
\end{tabular}

Note. In RNA expression metrics, I mainly focused on the small intestine, colon, stomach, and esophagus. In Protein expression, I mainly focused on the colon, stomach, and esophagus. The overall score is defined as the summation of RNA score and protein score.

Table 3. RNA and protein expression levels in the human brain

\begin{tabular}{|c|c|c|c|c|c|}
\hline & \multicolumn{3}{|c|}{ RNA expression metrics } & \multirow[b]{2}{*}{$\begin{array}{c}\text { Protein } \\
\text { expression score }\end{array}$} & \multirow[b]{2}{*}{$\begin{array}{c}\text { Overall } \\
\text { Average score }\end{array}$} \\
\hline & $\begin{array}{l}\text { RNA-seq } \\
\text { score }\end{array}$ & Microarray score & $\begin{array}{c}\text { SAGE } \\
\text { score }\end{array}$ & & \\
\hline SLC6A4 & 2 & 2 & 1 & 1 & 1.67 \\
\hline GRIK4 & 3 & 2 & 1 & 1 & 1.75 \\
\hline BDNF & 3 & 2 & 1 & 1 & 2 \\
\hline FKBP5 & 3 & 2 & 2 & 2 & 2.25 \\
\hline COMT & 3 & 3 & 3 & 3 & 3 \\
\hline $\mathrm{KCNK} 2$ & 3 & 2 & 3 & 1 & 2.67 \\
\hline GSK3B & 3 & 2 & 2 & 2.5 & 2.38 \\
\hline CRHBP & 3 & 2 & 1 & 1.5 & 1.88 \\
\hline IGF-1 & 2 & 2 & 1 & 1.25 & 1.75 \\
\hline IL1beta & 3 & 2 & 2 & 1 & 2 \\
\hline IL6 & 3 & 2 & 2 & 1 & 2 \\
\hline TNF Alpha & 2 & 2 & 2 & 1 & 1.75 \\
\hline
\end{tabular}

Note. Expression in the Brain. In RNA expression metrics, I mainly focused on the brain. In Protein expression, I mainly focused on the brain, brain fetal, frontal cortex, and cerebral cortex.

In all genes, these well-known genes have some extent of cerebral expressions, and they all show expression in the digestive system (Table 2). Comparing the average of the expression shown in the brain and the digestive system, the expression in the digestive system has an average of 1.81 , and the expression in the brain has an average of 2.1. We can conclude that the expression in the brain has a higher expression comparing the digestive system. 
Based on the relative expression level of all 12 known MDD related genes (Table 2 and 3), I realized that FKBP5, COMT, and GSK3B are most likely to be associated with brain-gut axis based on the expression analysis, since their expression levels are always among the highest in the brain and in the digestive system. I then searched the molecular and functional information of these 3 genes in the GeneCards database.

\section{FKBP5}

FKBP Prolyl Isomerase 5(FKBP5) is a member from the immunophilin protein family and it plays a role in immune regulation and basic cellular processes involved in protein folding and transport. Some related diseases that are related to FKBP5 are MDD and Asthma. FKBP5 plays a role in the risk of Dajor Depressive Disorder (MDD), response to treatment, and changes in brain regions [29].

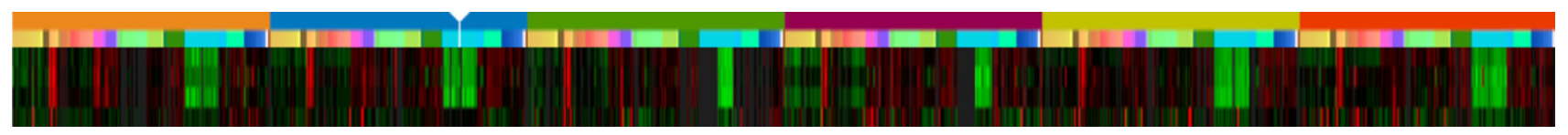

Fig. 4. Human Microarray for FKBP5.

It shows the Human expression in FKBP5. The red represents high expression and green represents low expression. The color shown in the first row of this figure represents different donors. It is hard to identify which tissue shows high expression all the time since there are many gene symbols and the expression is different for different donors.
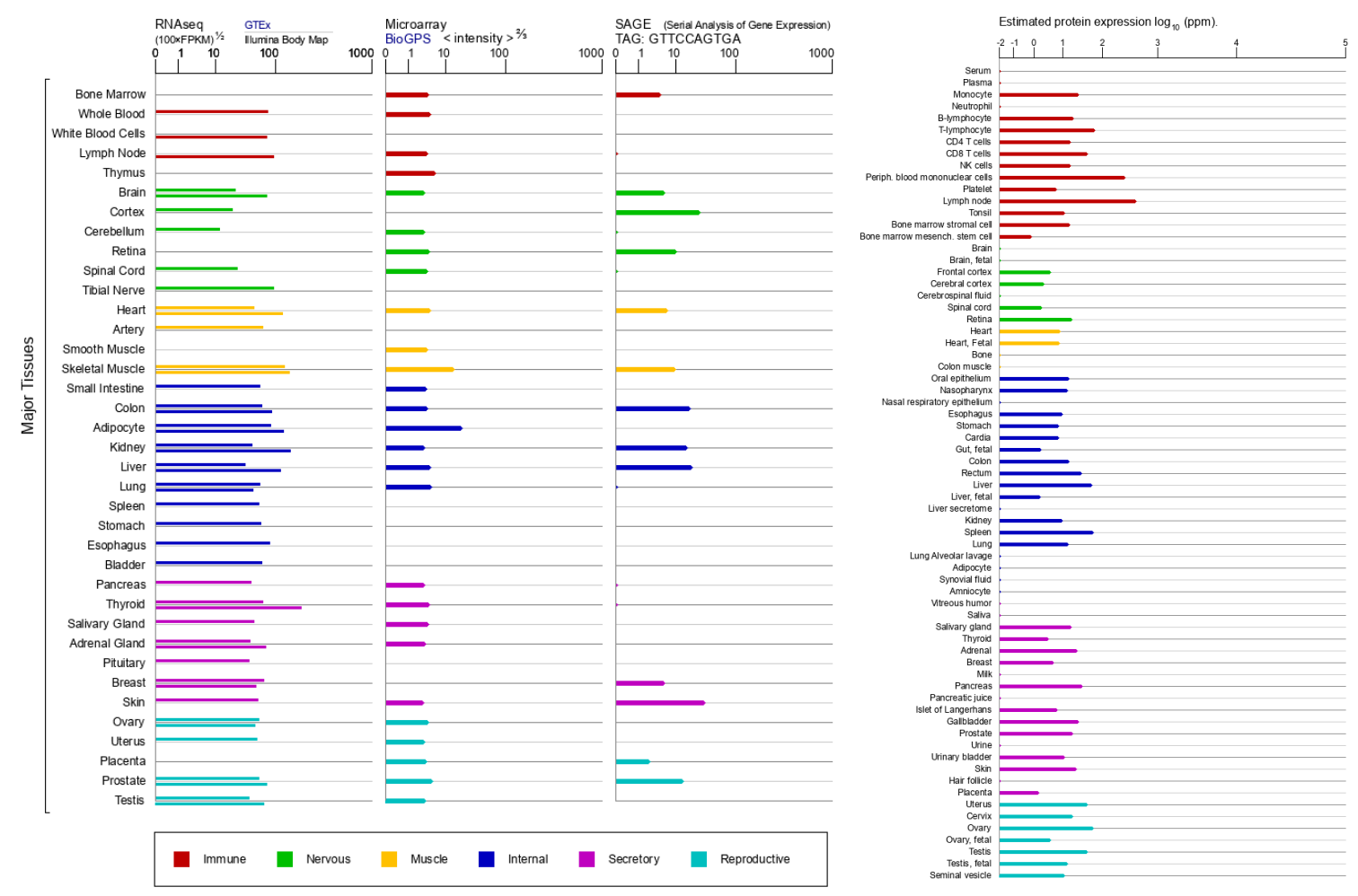

Fig. 5. Human RNA expression in FKBP5.

From the data, we can determine that the brain is expressing at an average expression for all three expressions.
From the data, the brain did not show a very high expression, however, it is still maintaining at the average. 
A.

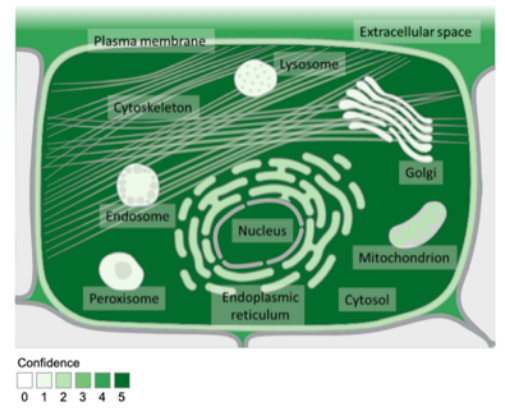

\begin{tabular}{|l|c|}
\hline Compartment & Confidence \\
\hline nucleus & 5 \\
\hline cytosol & 5 \\
\hline extracellular & 4 \\
\hline plasma membrane & 2 \\
\hline cytoskeleton & 2 \\
\hline mitochondrion & 2 \\
\hline endoplasmic reticulum & 2 \\
\hline peroxisome & 1 \\
\hline endosome & 1 \\
\hline lysosome & 1 \\
\hline golgi apparatus & 1 \\
\hline
\end{tabular}

B.

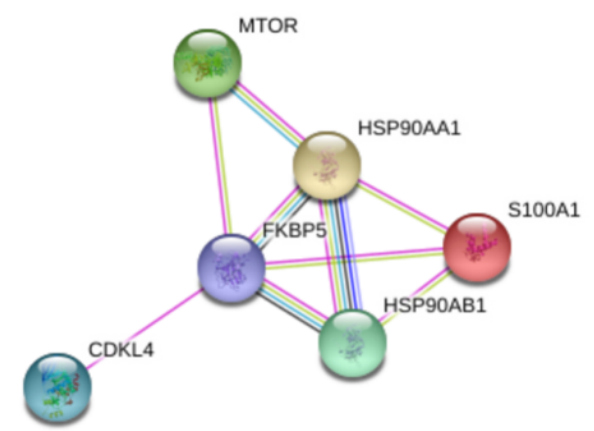

Fig. 6. Information about the localization and interacting proteins for FKBP5.

A. Localization for FKBP5. From this screenshot, we can explore that FKBP5 is most confident living in the nucleus and cytosol. B. Pathway for FKBP5. The screenshot presents some associated genes with FKBP5(CDKL4, HSP90AA1, HSP90AB1, MTOR, S100A1).

\section{COMT}

COMT (Catechol-O-Methyltransferase) transfers methyl groups from S-adenosylmethionine to catecholamines. COMT is also an important term in curing for hypertension, hypertension and Parkinson's disease. There was two types found in the COMT tissues, one is soluble (S-COMT), and the other is membrane bound form (MB-COMT). Diseases that are related to COMT are schizophrenia and panic disorder 1. COMT will influence the level of dopamine, and dopamine plays a very important role in psychiatric disorder, which indirectly proves that COMT is related to psychiatric disorders [30].

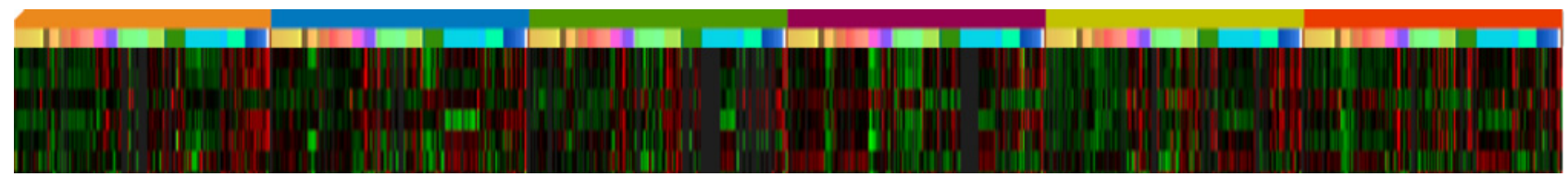

Fig. 7. Human Microarray for COMT.

It shows the human expression in COMT. The red represents high expression and green represents low expression. The colour shown in the first row of this figure represents different donors. It is hard to identify

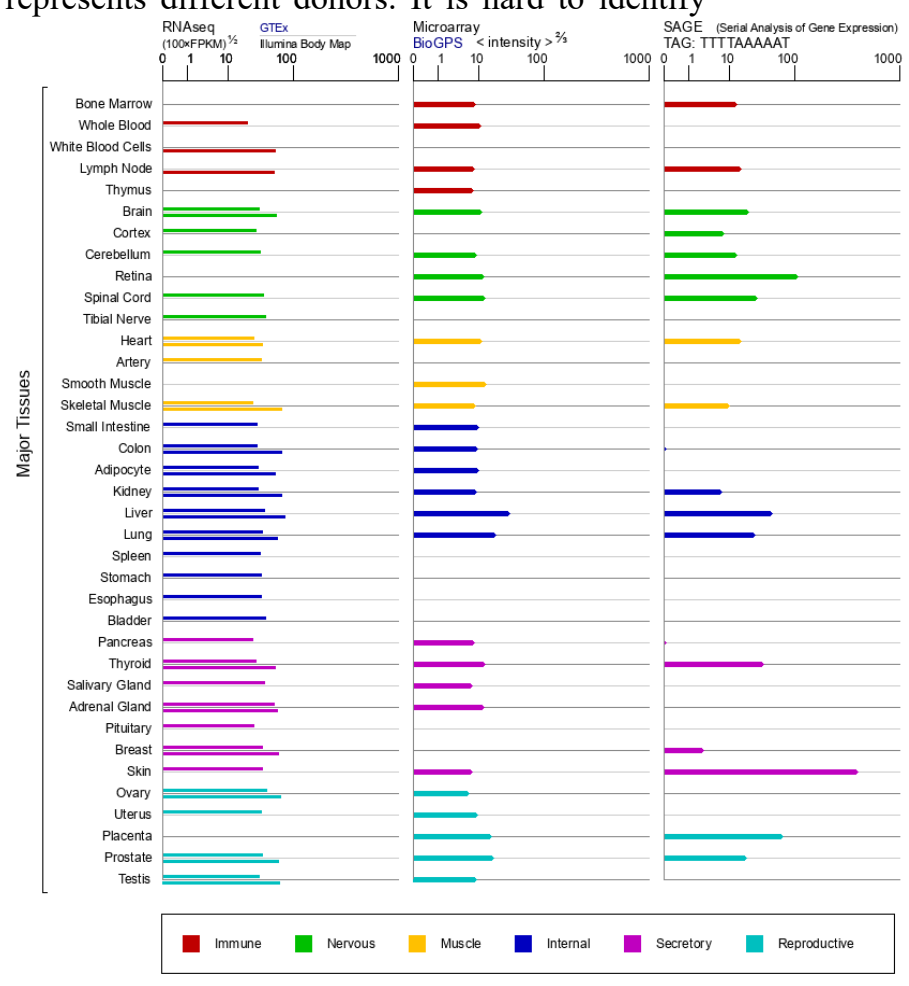

which tissue shows high expression all the time since there are many gene symbols and the expression is different for different donors.

Fig. 8. Human RNA expression in COMT. 
The data shows that the Nervous area was expressing at a very average rate in the RNA expression.

A.

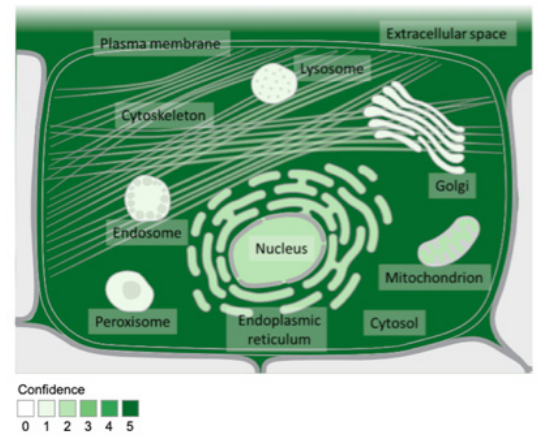

\begin{tabular}{|l|c|}
\hline Compartment & Confidence \\
\hline plasma membrane & 5 \\
\hline extracellular & 5 \\
\hline cytosol & 5 \\
\hline cytoskeleton & 2 \\
\hline mitochondrion & 2 \\
\hline nucleus & 2 \\
\hline endoplasmic reticulum & 2 \\
\hline peroxisome & 1 \\
\hline endosome & 1 \\
\hline lysosome & 1 \\
\hline golgi apparatus & 1 \\
\hline
\end{tabular}

In this data, the brain section also shows an expression at an average rate.

B.

Fig. 9. Information about the localization and interacting proteins for COMT.

A. Localization for COMT. The screenshot shows that COMT is most confident staying at plasma membrane, extracellular and cytosol.

B. Pathway for COMT. Some pathways for COMT are ACE, FGF2, RIN1 and PPA2.

\section{GSK3B}

GSK3B (Glycogen Synthase Kinase 3 Beta) is involved in energy metabolism, endoplasmic reticulum stress, inflammation and is glucose homeostasis negative regulator. Some related diseases are Bipolar Disorder and Alzheimer Disease.

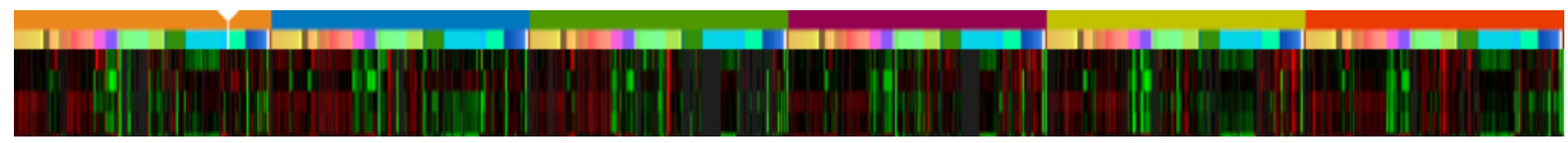

Fig. 10. Human Microarray for GSK3B.

It shows the human expression in GSK3B. The red represents high expression and green represents low expression. The colour shown in the first row of this figure represents different donors. It is hard to identify

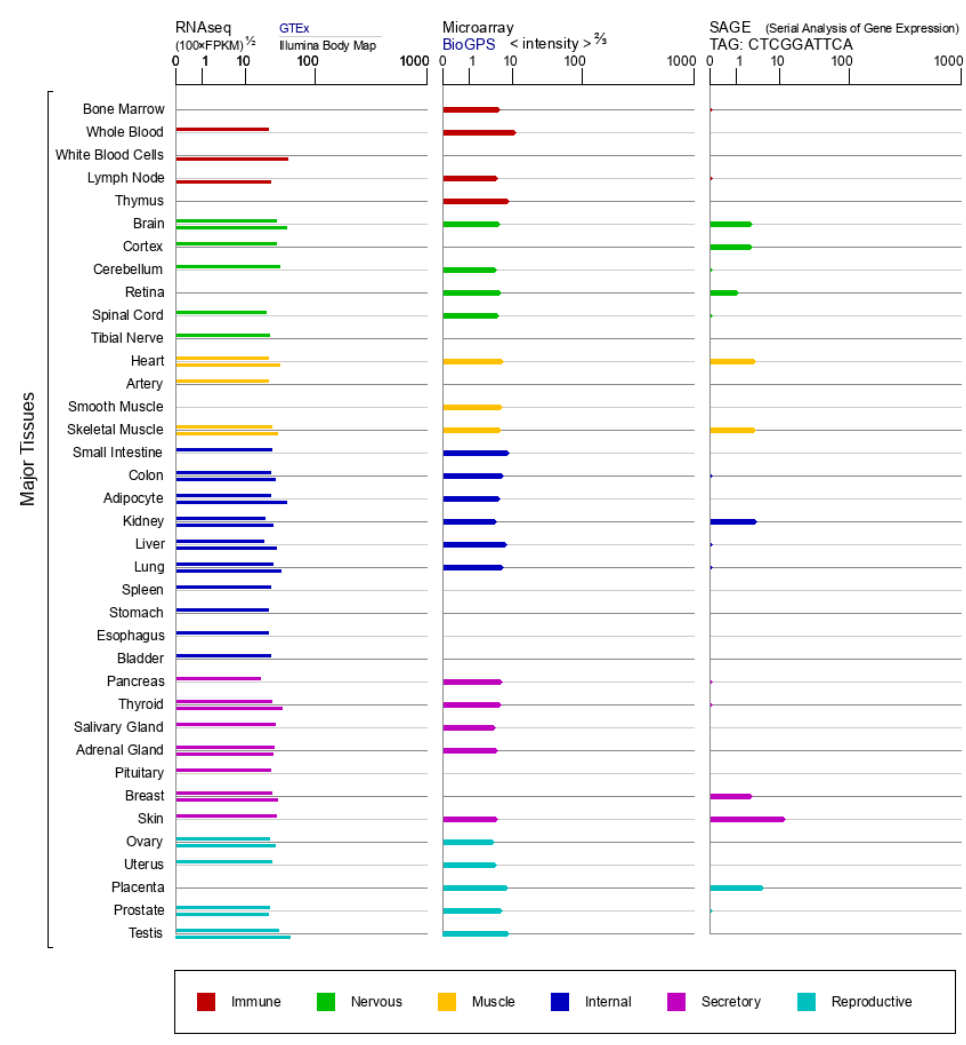

which tissue shows high expression all the time since there are many gene symbols and the expression is different for different donors.

Fig. 11. RNA expression in GSK3B.

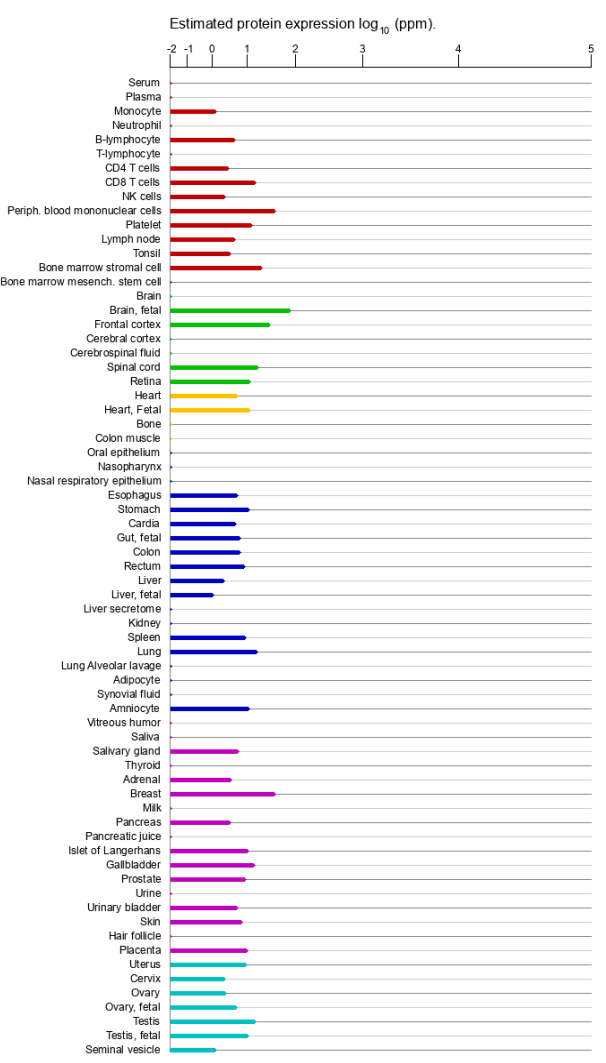


The data shows that the Nervous area was expressing at a very average rate in the RNA expression, and it is also expressed in SAGE where not a lot of tissues shows high expression.

A.

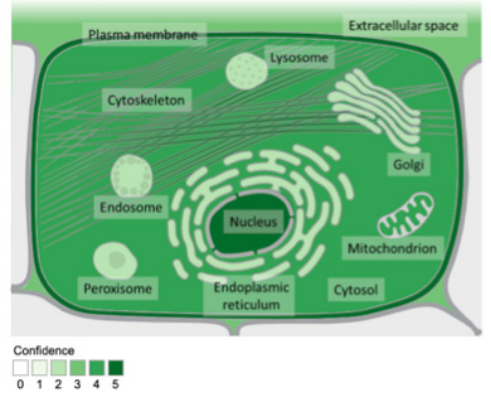

The brain fetal and frontal cortex shows a high expression in the protein expression, but the brain and cerebral cortex expression is very low.

Fig. 12. Information about the localization and interacting proteins for GSK3B.

A. Localization for GSK3B. The screenshot shows that plasma membrane, cytoskeleton and nucleus are all the confidence area for GSK3B to stay.

B. Pathway for GSK3B. From the screenshot, we can explore that AKT1, AKT3, AXIN1, FRAT1 and MAP2K7re all associated with GSK3B.

\subsubsection{Genes identified in GWAS of MDD}

In section 1, I already find several genes that might be linked to gut microbiota, however, the 12 genes I screened from only represent a very tiny proportion of the human genome. Therefore, in order to find more genes that might be related to MDD and gut microbiota, I employed the GWAS data from a recent published article which identified 44 genetic loci whose mutations are associated with higher risks of MDD [27]. These GWAS genes has helped me to narrow down the candidate gene list by filtering out genes unrelated to MDD from genome wide data. Starting from these data, I can further discover the genes that are also related to gut microbiota.

First, in order to simplify my computational screen, I narrowed down the list of 44 genetic loci, which has 68 genes, to 53 genes by only including the protein-coding genes (Table 4). To explore the possibility of these 53 genes in brain-gut axis, I further researched through Genecards about their expression levels in Small Intestine, Colon, Stomach, and Esophagus. When measuring, I employed the same metrics of RNA and protein expression as section 1 (Table 2).

Next, I set a threshold for each expression score to reduce the genes that show high expression. For the RNA-seq score, I set the threshold to be 12; for the Microarray score, I set the threshold to be 4; for the SAGE score, I set the threshold to be 1 , and for Protein expression score, I set the threshold to be 6 . Only the gene that has all scores above the threshold for each data will be involved. These genes will be presented with its average of RNA-seq, Microarray, SAGE, and Protein expression score (Table 5).

Table 4. Protein Coding Genes existed in GWAS

\begin{tabular}{cccccc}
\hline Gene Context & $\begin{array}{c}\text { Chromosome } \\
\text { No. }\end{array}$ & Region [27] & SNP & Location (bp) & P value \\
\hline RERE & 1 & $8.390-8.895$ & rs159963 & $8,504,421$ & $3.2 \times 10-8$ \\
SLC45A1 & 1 & $8.390-8.895$ & rs159963 & $8,504,421$ & $3.2 \times 10-8$ \\
NEGR1 & 1 & $72.511-73.059$ & rs1432639 & 72.813 .218 & $4.6 \times 10-15$ \\
DENND1B & 1 & $197.343-197.864$ & rs9427672 & $197,754,741$ & $3.1 \times 10-8$ \\
VRK2 & 2 & $57.765-58.485$ & rs11682175 & $57,987,593$ & $4.7 \times 10-9$ \\
NR4A2 & 2 & $157.978-157.464$ & rs1226412 & $157,111,313$ & $2.4 \times 10-8$ \\
GPD2 & 2 & $157.978-157.464$ & rs1226412 & $157,111,313$ & $2.4 \times 10-8$ \\
TOPAZ1 & 3 & $44.222-44.997$ & chr3_44287760_1 & $44,287,760$ & $4.6 \times 10-8$ \\
TCAIM & 3 & $44.222-44.997$ & chr3_44287760_1 & $44,287,760$ & $4.6 \times 10-8$ \\
\hline
\end{tabular}




\begin{tabular}{|c|c|c|c|c|c|}
\hline ZNF445 & 3 & $44.222-44.997$ & chr3_44287760_1 & $44,287,760$ & $4.6 \times 10-8$ \\
\hline RSRC1 & 3 & $157.616-158.354$ & rs 7430565 & $158,107,180$ & $2.9 \times 10-9$ \\
\hline MLF1 & 3 & $157.616-158.354$ & rs 7430565 & $158,107,180$ & $2.9 \times 10-9$ \\
\hline SLC30A9 & 4 & $41.880-42.189$ & rs34215985 & $42,047,778$ & $3.1 \times 10-9$ \\
\hline DCAF4L1 & 4 & $41.880-42.189$ & rs34215985 & $42,047,778$ & $3.1 \times 10-9$ \\
\hline MEF2C & 5 & $87.443-88.244$ & chr5_87992715_1 & $87,992,715$ & $7.9 \times 10-11$ \\
\hline TENM2 & 5 & $166.977-167.056$ & rs4869056 & $166,992,078$ & $6.8 \times 10-9$ \\
\hline FBXL4 & 6 & $99.335-99.662$ & rs9402472 & $99,566,521$ & $2.8 \times 10-8$ \\
\hline TMEM106B & 7 & $12.154-12.381$ & rs10950398 & $12,264,871$ & $2.6 \times 10-8$ \\
\hline VWDE & 7 & $12.154-12.381$ & rs10950398 & $12,264,871$ & $2.6 \times 10-8$ \\
\hline PUM3 & 9 & 2.919-3.009 & rs 1354115 & $2,983,774$ & $2.4 \times 10-8$ \\
\hline ASTN2 & 9 & $119.675-119.767$ & rs 7856424 & $119,733,595$ & $8.5 \times 10-9$ \\
\hline DENND1A & 9 & $126.292-126.735$ & rs 7029033 & $126,682,068$ & $2.7 \times 10-8$ \\
\hline LHX2 & 9 & $126.292-126.735$ & rs7029033 & $126,682,068$ & $2.7 \times 10-8$ \\
\hline SORCS3 & 10 & 106.397-106.904 & rs61867293 & $106,563,924$ & $7.0 \times 10-10$ \\
\hline ELP4 & 11 & $31.121-31.859$ & rs 1806153 & $31,850,105$ & $1.2 \times 10-9$ \\
\hline PAX6 & 11 & $31.121-31.859$ & rs1806153 & $31,850,105$ & $1.2 \times 10-9$ \\
\hline SOX5 & 12 & $23.924-24.052$ & rs 4074723 & $23,947,747$ & $3.1 \times 10-8$ \\
\hline ENOX1 & 13 & $44.237-44.545$ & rs4143229 & $44,327,799$ & $2.5 \times 10-8$ \\
\hline LACC1 & 13 & $44.237-44.545$ & rs4143229 & $44,327,799$ & $2.5 \times 10-8$ \\
\hline CCDC122 & 13 & $44.237-44.545$ & rs4143229 & $44,327,799$ & $2.5 \times 10-8$ \\
\hline OLFM4 & 13 & $53.605-54.057$ & rs 12552 & $53,625,781$ & $6.1 \times 10-19$ \\
\hline LRFN5 & 14 & $41.941-42.320$ & rs4904738 & $42,179,732$ & $2.6 \times 10-9$ \\
\hline SYNE2 & 14 & $64.613-64.878$ & rs915057 & $64.686,207$ & $7.6 \times 10-10$ \\
\hline ESR2 & 14 & $64.613-64.878$ & rs915057 & $64.686,207$ & $7.6 \times 10-10$ \\
\hline DLST & 14 & $75.063-75.398$ & chr14_75356855_1 & $75,356,855$ & $3.8 \times 10-9$ \\
\hline PROX2 & 14 & $75.063-75.398$ & chr14_75356855_1 & $75,356,855$ & $3.8 \times 10-9$ \\
\hline RPS6KL1 & 14 & $75.063-75.398$ & chr14_75356855_1 & $75,356,855$ & $3.8 \times 10-9$ \\
\hline BAG5 & 14 & $103.828-104.174$ & rs10149470 & $104,017,953$ & $3.1 \times 10-9$ \\
\hline RBFOX1 & 16 & $6.288-6.347$ & rs 8063603 & $6,310,645$ & $6.9 \times 10-9$ \\
\hline RBFOX1 & 16 & $7.642-7.676$ & rs7198928 & $7,666,402$ & $1.0 \times 10-8$ \\
\hline SHISA9 & 16 & $13.022-13.119$ & rs7200826 & $13,066,833$ & $2.4 \times 10-8$ \\
\hline CPPED1 & 16 & $13.022-13.119$ & rs7200826 & $13,066,833$ & $2.4 \times 10-8$ \\
\hline PMFBP1 & 16 & $71.631-72.849$ & rs11643192 & $72,214,276$ & $3.4 \times 10-8$ \\
\hline DHX38 & 16 & $71.631-72.849$ & rs11643192 & $72,214,276$ & $3.4 \times 10-8$ \\
\hline CRYBA1 & 17 & $27.345-28.419$ & rs17727765 & $27,576,962$ & $8.5 \times 10-9$ \\
\hline MYO18A & 17 & $27.345-28.419$ & rs17727765 & $27,576,962$ & $8.5 \times 10-9$ \\
\hline NUFIP2 & 17 & $27.345-28.419$ & rs 17727765 & $27,576,962$ & $8.5 \times 10-9$ \\
\hline
\end{tabular}




\begin{tabular}{cccccc}
\hline DCC & 18 & $50.358-50.958$ & rs11663393 & $50,614,732$ & $1.6 \times 10-8$ \\
RAB27B & 18 & $51.974-52.552$ & rs 183288 & $52,517,906$ & $2.6 \times 10-8$ \\
CCDC68 & 18 & $51.974-52.552$ & rs183288 & $52,517,906$ & $2.6 \times 10-8$ \\
TCF4 & 18 & $52.860-53.268$ & rs 12958048 & $53,101,598$ & $3.6 \times 10-11$ \\
L3MBTL2 & 22 & $40.818-42.216$ & rs5758265 & $41,617,897$ & $7.6 \times 10-9$ \\
CHADL & 22 & $40.818-42.216$ & rs5758265 & $41,617,897$ & $7.6 \times 10-9$
\end{tabular}

Table 5. RNA and protein expression levels in the human digestive system

\begin{tabular}{|c|c|c|c|c|c|}
\hline & \multicolumn{3}{|c|}{ RNA expression metrics } & Protein expression & $\begin{array}{c}\text { Overall } \\
\text { score }\end{array}$ \\
\cline { 2 - 4 } & RNA-seq score & Microarray score & SAGE score & 4 & 2.75 \\
\hline GPD2 & 3 & 2 & 2 & 2.33 & 2.33 \\
\hline SLC30A9 & 3 & 3 & 1 & 2 & 2 \\
\hline OLFM4 & 3 & 2 & 2 & 3.67 & 3.23 \\
\hline DLST & 3.25 & 4 & 2 & 4.67 & 2.92 \\
\hline BAG5 & 3 & 2 & 1 & 3.33 & 2.58 \\
\hline CPPED1 & 3 & 3 & 1 & 4 & 2.5 \\
\hline DHX38 & 3 & 2 & 3 & 2 & 2.5 \\
\hline RAB27B & 3 & 2 & 1 & 4 & \\
\hline
\end{tabular}

Note. Table 5 shows the average expressions of the genes that are above threshold in the field of Small Intestine, Colon, Stomach, and Esophagus.

Table 6. RNA and protein expression levels in the human brain

\begin{tabular}{|c|c|c|c|c|c|}
\hline & \multicolumn{3}{|c|}{ RNA expression metrics } & Protein expression & $\begin{array}{c}\text { Overall } \\
\text { score }\end{array}$ \\
\cline { 2 - 4 } & RNA-seq score & Microarray score & SAGE score & 4.25 & 6 \\
\hline GPD2 & 3 & 2 & 2 & 1.5 & 3.75 \\
\hline SLC30A9 & 3 & 3 & 3 & 1 & 2.5 \\
\hline OLFM4 & 3 & 2 & 1 & 1.25 & 1.56 \\
\hline DLST & 2 & 2 & 1 & 2 & 2.25 \\
\hline BAG5 & 3 & 2 & 2 & 3 & 2.5 \\
\hline CPPED1 & 3 & 2 & 2 & 1.5 & 2.13 \\
\hline DHX38 & 3 & 2 & 2 & 3 & 2.5 \\
\hline RAB27B & 3 & 2 & 2 & 2 & \\
\hline
\end{tabular}

Note. Table 6 shows the average expression for the genes that are above threshold genes expression in the brain.

Then, in order to narrow down the genes, I collected the expression for the genes showed in the brain after screening out the above threshold genes. Base on the Overall protein score showed in the data Expressions of the genes that are above threshold in the field of Small Intestine, Colon, Stomach and Esophagus, I found out 4 most interesting genes for further research: OLFM4, DLST, CPPED1, RAB27B.

\section{OLFM4}

Olfactomedin 4 (OLFM4) gene encodes an extracellular matrix protein that is believed to be antiapoptotic. The gene is selectively highly expressed in colonic epithelium and moderately expressed in several nervous tissue (including cerebral cortex, cerebellum, retina, and spinal cord) (Figure 13). Some disease that is related to OLFM4 are Pancreatic Cancer and Ovary Serous Adenocarcinoma. OLFM4 is related to many digestive diseases. In recent study about Helicobacter pylori (H. pylori) infection of gastric mucosa, it shows an increase in the expression of OLFM4. OLFM4 plays an important role in the mucosal defense of inflammatory bowel disease [31].

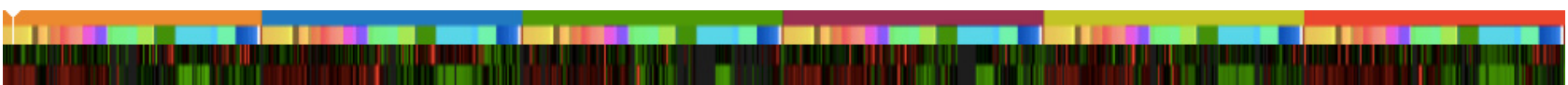

Fig. 13. Human microarray data of OLFM4 from Allen Brain Atlas.

This screenshot is a picture showing the gene OLFM4. In the screenshot, the red symbolized as a higher activity in genes of interest. The colour shown in the first row of this figure represents different donors. In all donors, amygdalohippocampal transition zone (ATZ) and tail of the caudate nucleus (TCd) all shows red on the picture, which means in these parts OLFM4 shows active. 

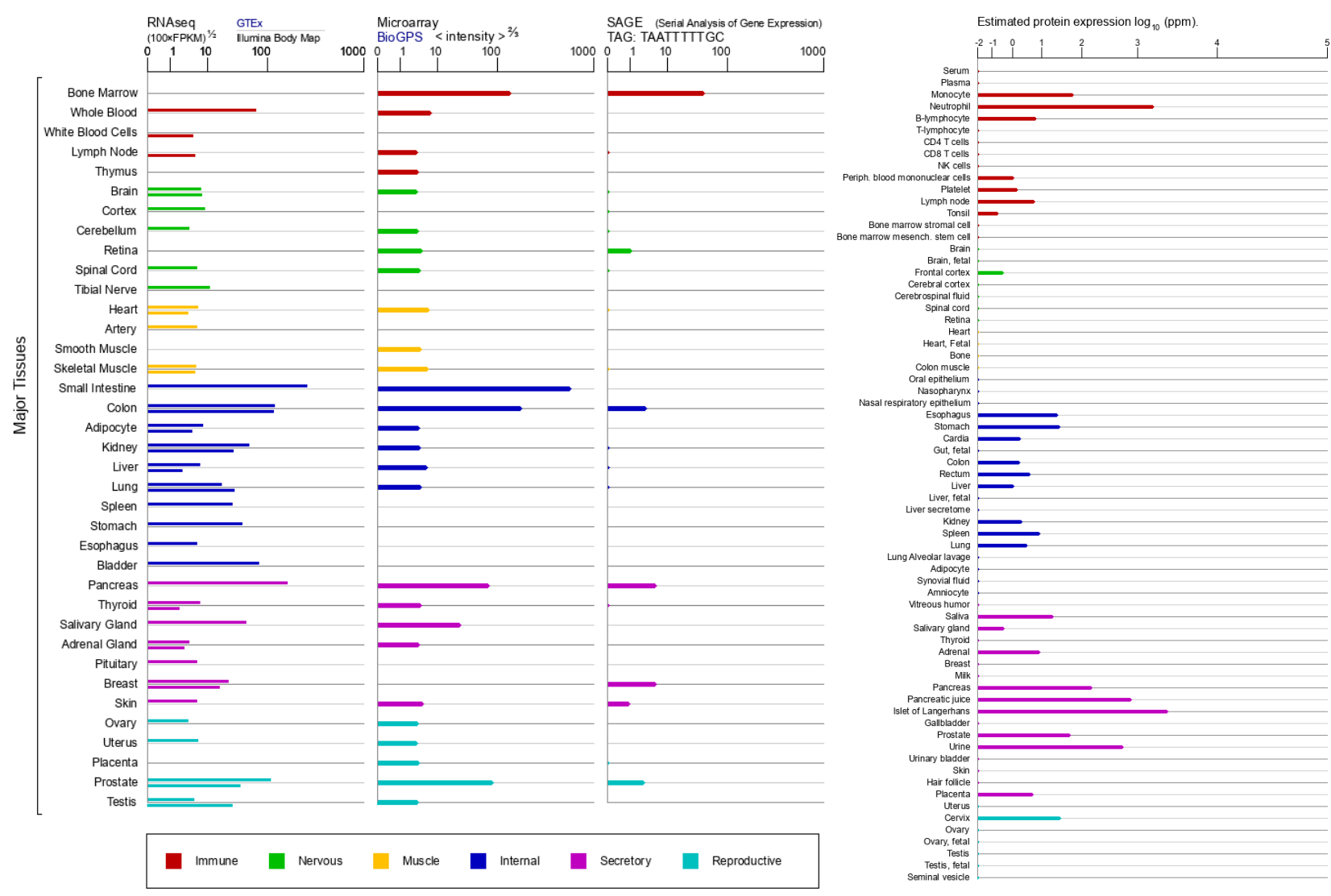

Fig. 14. Human RNA expression of OLFM4.

From the graph, we can identify that the internal section is showing a very high expression, representing that OLMF4 did affect some parts in gut microbiota.

A.

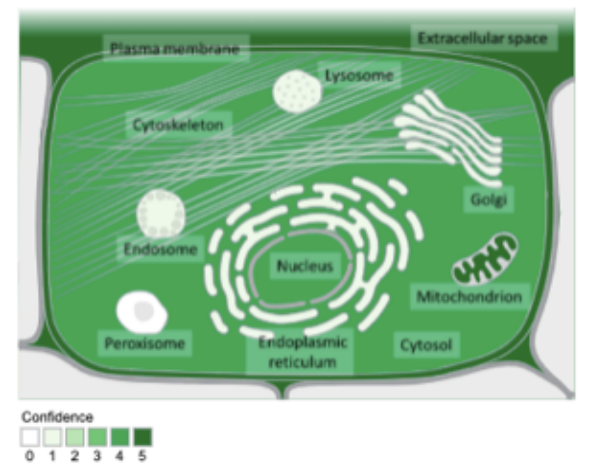

From the graph, we can explore that internal part didn't show a very high expression, but its expression is still staying at the average.

B.

\begin{tabular}{|l|c|}
\hline Compartment & Confidence \\
\hline plasma membrane & 5 \\
\hline extracellular & 5 \\
\hline mitochondrion & 5 \\
\hline nucleus & 4 \\
\hline cytosol & 4 \\
\hline cytoskeleton & 1 \\
\hline endoplasmic reticulum & 1 \\
\hline endosome & 1 \\
\hline lysosome & 1 \\
\hline golgi apparatus & 1 \\
\hline
\end{tabular}

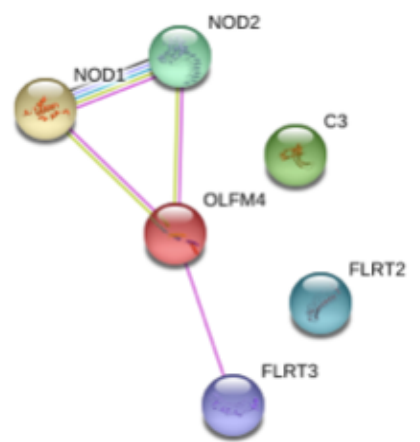

Fig. 15. Information about the localization and interacting proteins for OLFM4. A. Different compartment and the confidence rate for OLFM4 to live.

B. The top 5 STRING interaction network for OLFM4 (FLRT3, NOD1, NOD2)

\section{RAB27B}

RAB27B is in the RAS Oncogene Family. RAB27B can be related to diseases including Griscelli Syndrome and Piebald Trait. Some superpathways includes Response to elevated platelet cytosolic $\mathrm{Ca} 2+$, Vesiclemediated transport and Metabolism of protein.

The RNA of RAB27B shows multiple major tissues active, however, in the protein expression data, Brain and stomach are the highest expression in their lineages, which displays that gut-immune system-brain axis might play a role in RAB27B. In order to further understand that which part of the brain has high expression, I did a further analysis through Allen Brain Atlas, and it demonstrates that the frontal cortex, amygdala, diencephalon shows a high activeness. Frontal cortex and amygdala are all the brain parts that will show high activity in MDD, which further confirm that RAB27B might be related to MDD. 


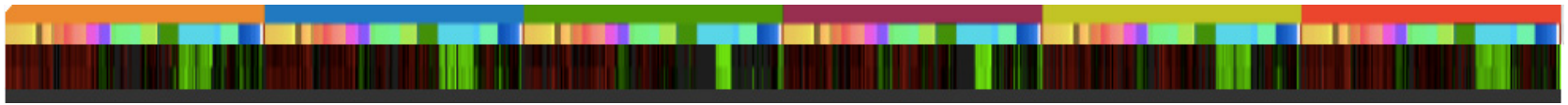

Fig. 16. Human microarray data of RAB27B.

The microarray data shows the activity in genes of interest indifferent parts of the brain. The red represents high activeness, and the green shows lower activeness. The color shown in the first row of this figure represents different donors. After comparing all donors with all activeness, Globus pallidus, internal segment in the basal ganglia, cingulum bundle in the white matter shows high activeness.
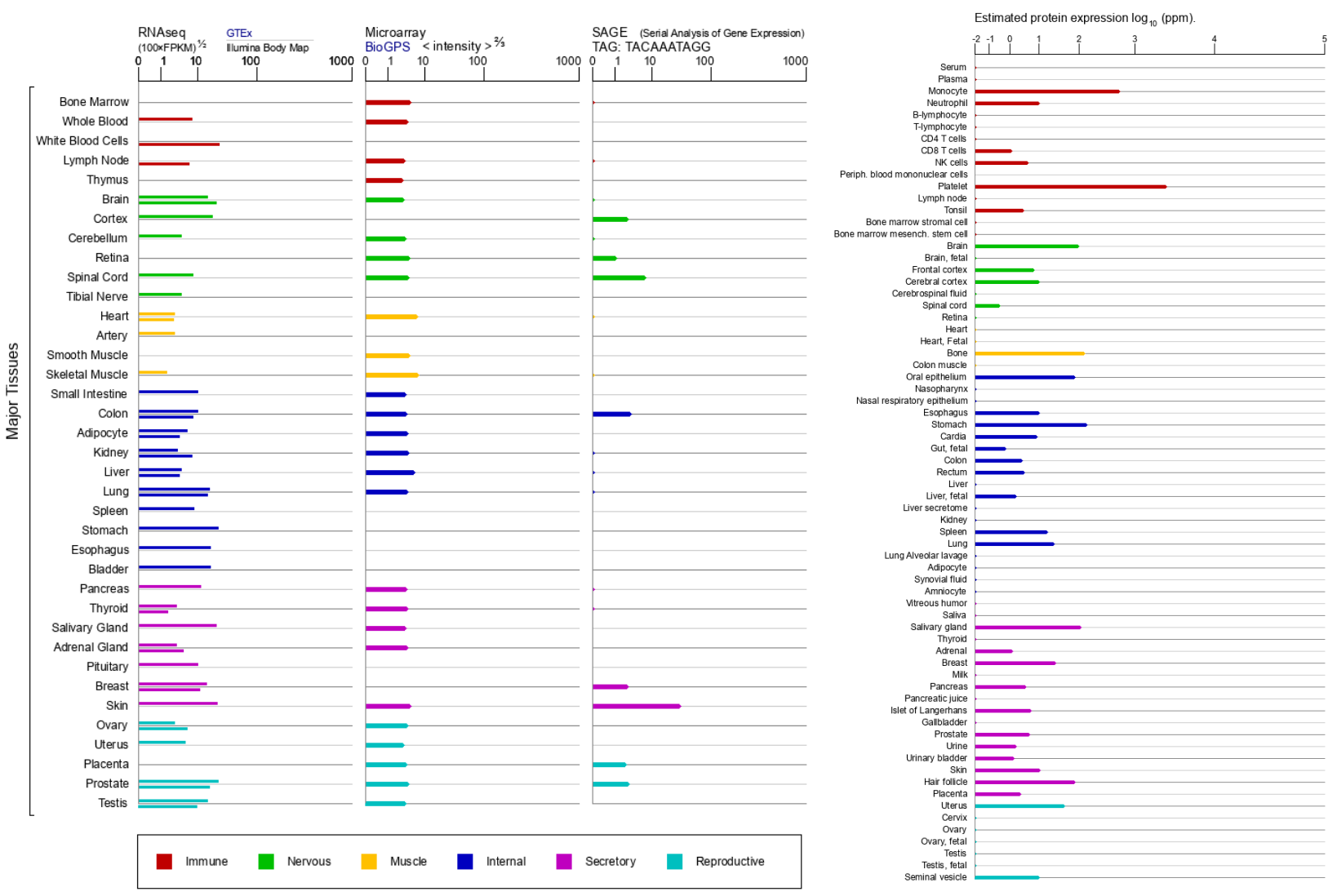

Fig. 17. RNA expression level of RAB27B.

Although brain and stomach both demonstrates high activity, mostly all the major tissues are active, therefore we cannot assure that this gene might contain the gut immune system brain axis.

A.

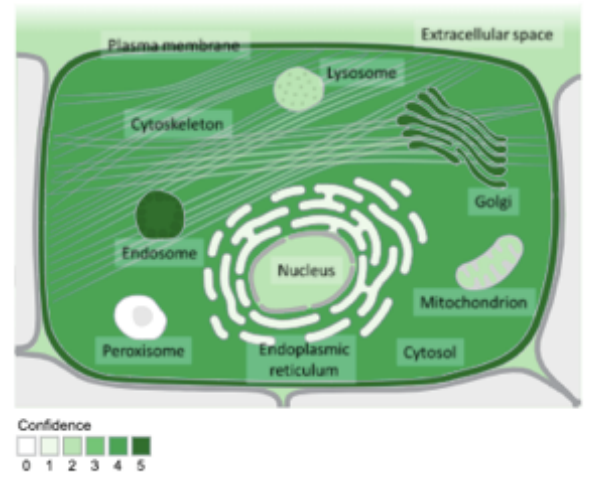

The data shows that Stomach and Brain are the highest in their lineage, which displays that the gut immune system brain axis might be involve in this gene.

Fig. 18. $T$ the localization and interacting proteins for RAB27B.

A. Different compartment and the confidence rate for RAB27B to live.

B. The top 5 STRING interaction network for RAB27B (DOC2A, DOC2B, MLPH, RPH3A, RPH3AL) 


\section{DLST}

Dihydrolipoamide S-Succinyltransferase (DLST) is a member of the 2-oxoacid dehydrogenase family. Some associated diseases with DLST are Paragangliomas 7 and Hereditary Paraganglioma-Pheochromocytoma Syndromes. In the Human T-ALL cell line, the symptom of a decreasing of cell viability and induction of apoptosis was caused by the RNAi inhibition of DLST, which also disrupted the TCA cycle [32]. The TCA cycle is affected by the young and mid-age MDD patients, which indirectly proved that DLST might be associated with MDD.

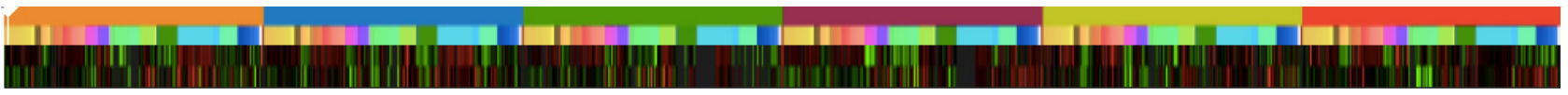

Fig. 19. Human microarray data of DLST.

The red represents high expression and the green represents low expression. The color shown in the first row of this figure represents different donors. In all donors, VI(Cb-VI), VIIA(Cb-VIIA), V(Cb-V), IV(Cb-
IV), Crus I (Cb-Crus I) and Crus II (Cb-Crus II) all shows high expression, which might show that these four structures play a very important role in DLST.
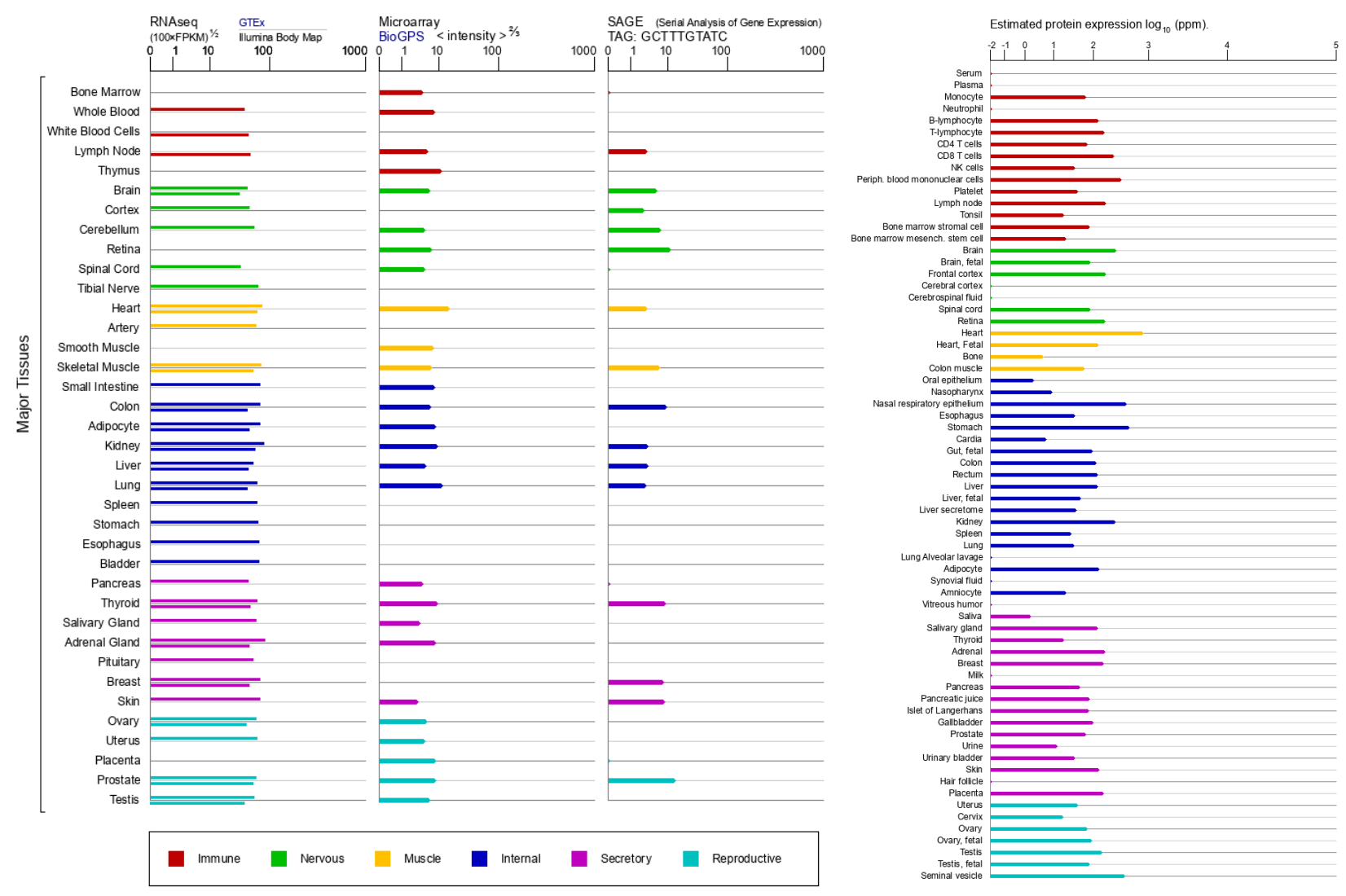

Fig. 20. RNA expression level of DLST.

In Figure 20, the expression for brain and the digestive system are mostly at the same level as other major tissues, but the expression appears for all three data, which might show that it is still significant in DLST.
Although all the tissues show expression, but we can still conclude that stomach, brain, and nasal respiratory epithelium shows a higher expression then other tissue. 
A.

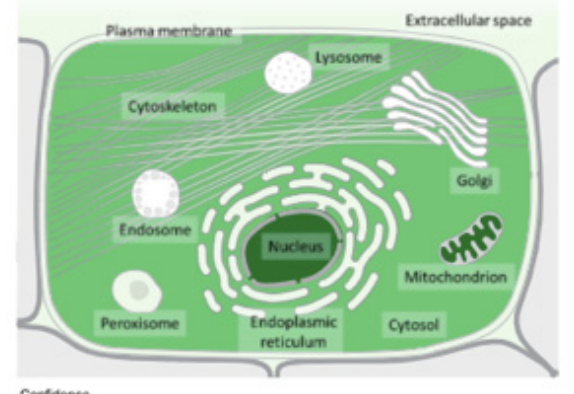

B.

\begin{tabular}{|l|c|}
\hline Compartment & Confidence \\
\hline mitochondrion & 5 \\
\hline nucleus & 5 \\
\hline cytosol & 3 \\
\hline plasma membrane & 1 \\
\hline extracellular & 1 \\
\hline cytoskeleton & 1 \\
\hline peroxisome & 1 \\
\hline endoplasmic reticulum & 1 \\
\hline
\end{tabular}

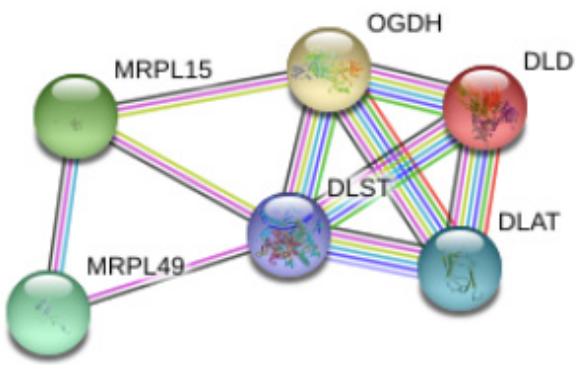

Fig. 21. T the localization and interacting proteins for DLST.

A. Different compartment and the confidence rate for DLST to live.

B. The top 5 STRING interaction network for DLST (DLAT, DLD, MRPL15, MRPL49, OGDH).

\section{CPPED1}

Calcineurin Like Phosphoesterase Domain Contaning 1 (CPPED1) blocks the cell cycle progression and promote the apoptosis of the cell. Some related diseases with CPPED1 are Trichostrongyloidiasis and Trichostrongylosis.

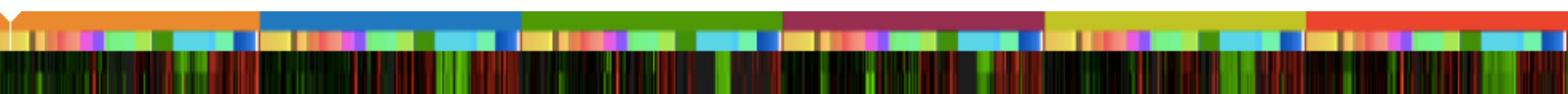

Fig. 22. Human microarray data of CPPED1.

The red represents high expression and the green represents low expression. The color shown in the first row of this figure represents different donors. In all donors, globus pallidus, internal segment (GPi), ventral

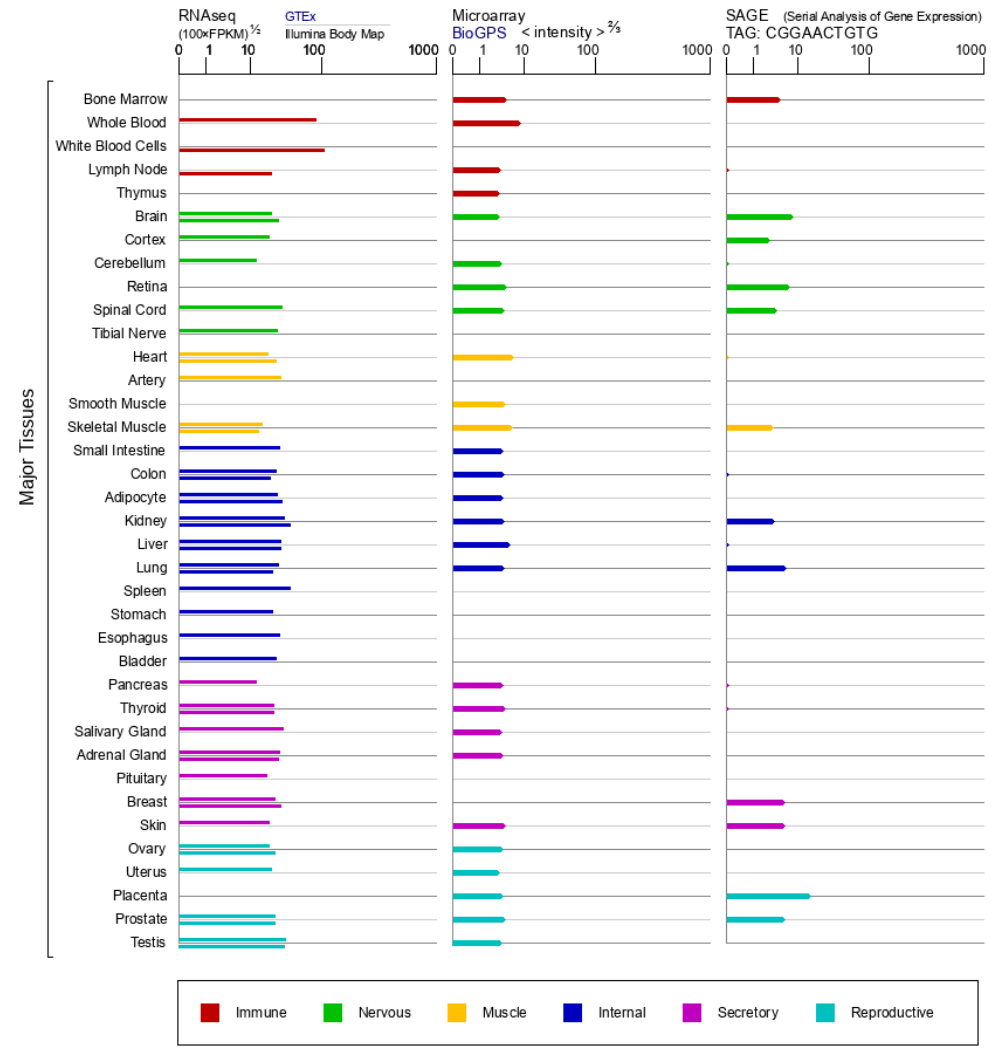

thalamus (VT) and corpus callosum(cc) all shows red, which represents high expression, which might show that these four structures play a very important role in DLST.

Fig. 23. RNA expression level of CPPED1.

In figure 23 , both brain and digestive system was at an average expression. 
In Figure 24, the brain didn't show a very high expression, but the digestive system shows a very high expression in the protein expression data.
A.

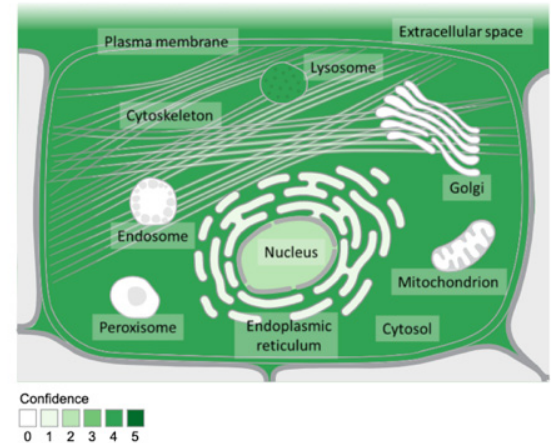

B.

\begin{tabular}{|l|c|}
\hline Compartment & Confidence \\
\hline plasma membrane & 4 \\
\hline extracellular & 4 \\
\hline cytosol & 4 \\
\hline lysosome & 4 \\
\hline nucleus & 2 \\
\hline cytoskeleton & 1 \\
\hline endoplasmic reticulum & 1 \\
\hline peroxisome & 0 \\
\hline
\end{tabular}

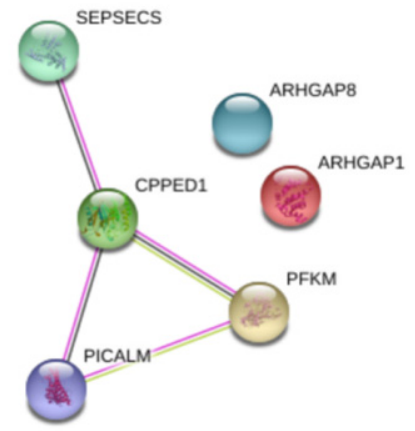

Fig. 24. $T$ the localization and interacting proteins for CPPED1.

A. Different compartment and the confidence rate for CPPED1 to live.

B. The top 5 STRING interaction network for CPPED1 (PFKM, PICALM, SEPSECS).

\subsection{Expression and Pathway Analysis of Identified Candidate Genes}

After exploring the 7 genes that might be highly related with MDD and gut Microbiota, I am going to further explore the pathways and their relations with gut microbiota.

\subsubsection{STRING Pathway Analysis of the Candidate Genes}

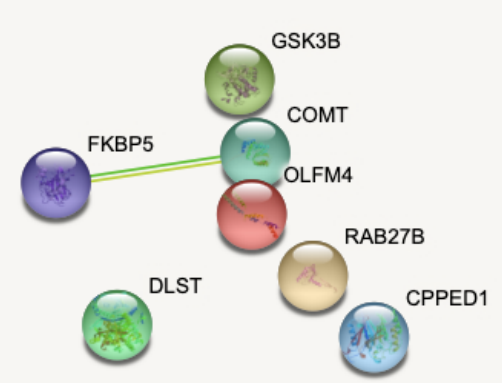

Fig. 25. Relationships between the 7 genes related to brain-gut

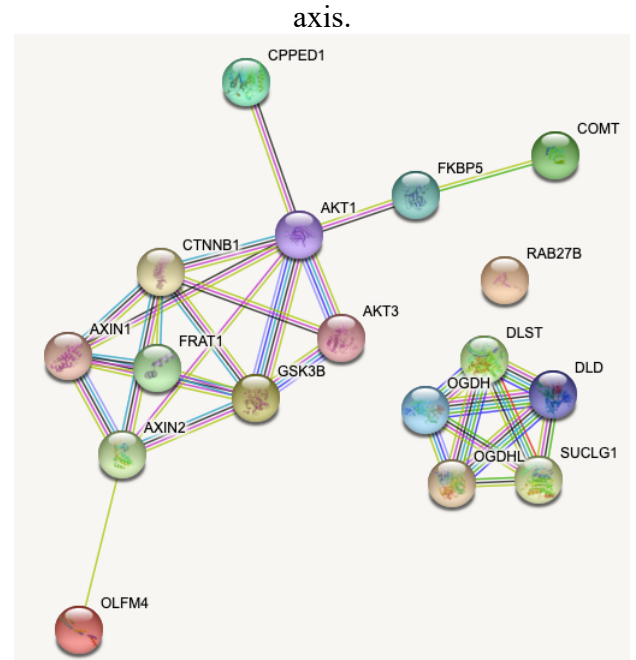

Fig. 26. Expansion of the relationships between the 7 genes related to brain-gut axis.
In Figure 26, it only shows the connection between the seven genes. And the only related genes are FKBP5 and COMT. These seven genes have no direct connections between each other. However, research methods for MDD are limited, some intermediate components may be missed. Therefore, I adjusted the settings by the STRING algorithm and expanded the molecular network to find more "node" genes. The expanded STRING network has demonstrated that (1) OLFM4 was connected through AXIN2, (2) DLST still did not show any connections, and (3) RAB27B has no connections with all 6 other genes all the time. Clearly, I found 2 central molecular pathways which are Wnt Signaling and citric acid cycle.

\section{Wnt Signaling Pathway}

The Wnt Signaling Pathway conducts the cell's migration, polarity, neural patterning, and other body parts in the embryo. When related to some antidepressant treatment, the content of wnt will increase [12]. Several evidences from animal models of depression, it shows that glycogen synthase kinase 3 beta (GSK3 3 ) plays a very important role in MDD [33]. Through a further research, it proves the hypothesis that the polymorphism in GSK3 $\beta$ plays a role in the susceptibility or expression of MDD, partly because it acts through the classic Wnt signaling pathway and related substrates [34].

Also, Wnt Signaling Pathway is also linked to gut microbiota. Recent studies showed Wnt signaling pathway is tightly linked to cancer; in colorectal cancer, it has most prominently been described [35]. We can infer that wnt signaling pathway might have some relationships with the digestive system through the secretion of the Wnt signaling. By all means, GSK3B shows a high expression in both the brain and the digestive system, and it is also linked to Wnt Signaling Pathway. It can be inferred that Through Wnt Signaling.

\section{Citric Acid cycle}

Citric Acid cycle is a central biochemical process in aerobic respiration which is conserved from bacteria to human beings. It is not surprising that citric acid cycle may link the metabolism of gut microbiota and their host. 
Some evidences from an article that focused on leukocyte tricarboxylic acid cycle and Bipolar Disorder show that mitochondrial dysfunction plays a role in the pathophysiology of bipolar disorder [27,36]. Genetic mutations on $\alpha$-ketoglutarate-dependent dioxygenase (FTO) leads to a higher average body mass index (BMI) higher risk of MDD [37].

\subsubsection{Gene Ontology (GO) analysis}

To further understand the molecular functions of the 7 candidate genes associated with both MDD and gut microbiota, I conducted the GO analysis. To avoid bias, I chose two different GO analysis tools: g:profiler and DAVID [23].

\section{1) GO analysis by gprofiler ( $R$ studio based)}

The g:profiler algorithm first provide a Mahattan-like plot which illustrates which GO categories the significant GO terms fall into. The Mahattan-like plot generated based on my 7 input candidate genes indicates one important GO term: catalytic activity (GO: 0003824) (Figure 27). The number of annotated GO terms in this gene list is relatively few, since only 7 genes have been discovered in my computational screen. Catalytic activity can be also named as enzyme activity in GO annotation. This result is consistent with the finding from STRING pathway analysis in which several enzymes of citric acid cycle are involved.

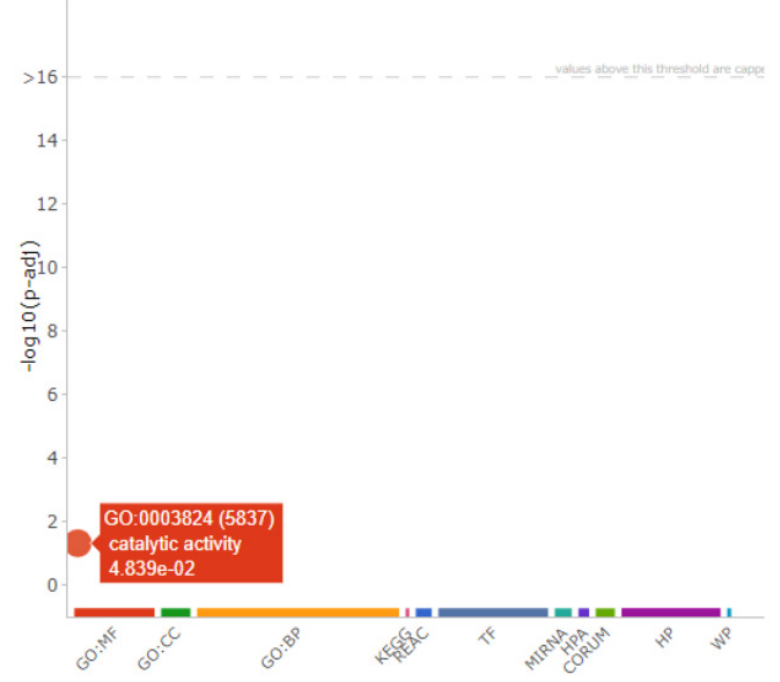

Fig. 27. Manhattan-like Plot from R-studio.
In the graph, GO:MF stands for Gene Ontology: Molecular Function; GO:CC stands for Gene Ontology: Cellular Component; GO:BP stands for Gene Ontology: Biological Process; KEGG stands for Kyoto Encyclopedia of Genes and Genomes; REAC stands for Restriction endonuclease analysis of chromosomal DNA; TF stands for Transcription Factor; MIRNA stands for MicroRNA; HPA stands for Human Protein Atlas; CORUM stands for the Comprehensive Resource of Mammalian Protein Complexes; HP stands for haptoglobin.

\subsubsection{DAVID}

DAVID provides the ability to perform functional analysis of large gene lists about biological meanings. By using DAVID, it enables a detailed gene list for functional analysis for 7 genes.

Table 7. DAVID Functional Annotation Chart

\begin{tabular}{|c|c|c|c|}
\hline GO Category & GO term & P value & Benjamin \\
\hline $\begin{array}{c}\text { GOTERM_CC__ } \\
\text { DIRECT }\end{array}$ & $\begin{array}{c}\text { extracellular } \\
\text { exosome }\end{array}$ & $2.4 \mathrm{E}-2$ & $2.9 \mathrm{E}-1$ \\
\hline
\end{tabular}

Note. Within these seven genes, there is only one GO category shown in DAVID, and its term is extracellular exosome.

After importing the 7 genes, DAVID presented a graph that shows the related GO Category, which is extracellular exosome. Exosome has the ability to processes synaptic plasticity, neuronal stress response, intercellular communication and neurogenesis in the brain, which is connected to the brain [38]. Also, studies confirmed by delivering antibacterial products, the secretion of exosomes to the top of the lumen will regulate the function of distant cells along the gastrointestinal tract or regulate the homeostasis of the gut microbiota [39]. 


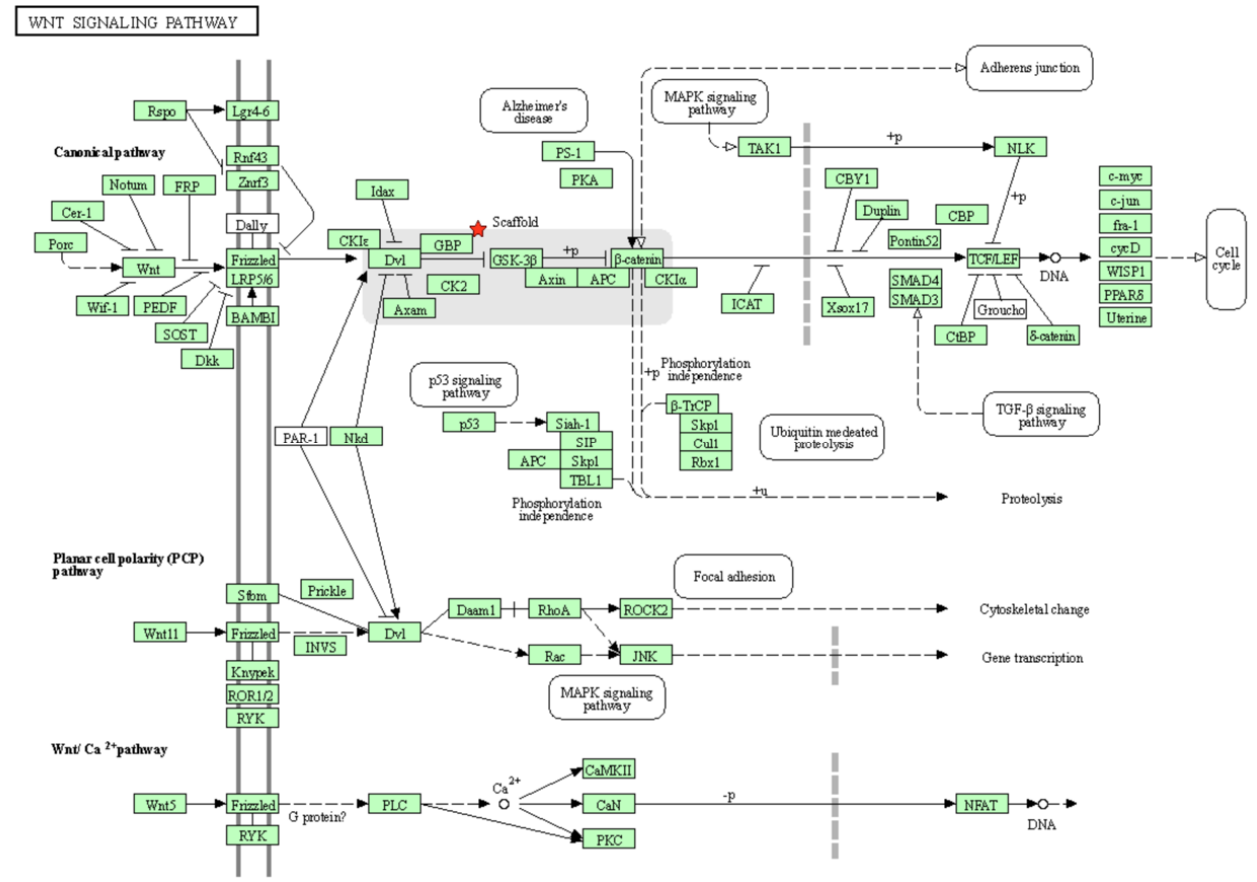

Fig. 28. Signaling Pathways identified by DAVID algorithm.

After giving the information about the 7 genes, it gives the WNT Signaling Pathway. In the pathway, the gene that appears is GSK3B. These results provide evidence in section 2.1, further acknowledged that the Wnt Signaling pathway is a pathway in these 7 genes.

\subsection{Gut Microbiome Analysis Based on the Screened Genes}

The 7 genes that was chosen has the highest expression shown in both the brain and the digestive system among all genes. However, the analysis presented previously has not yet shown any relationship with the gut microbiota. Therefore, I am going to further explore the relationship between gut microbiota through a gut microbiome analysis.

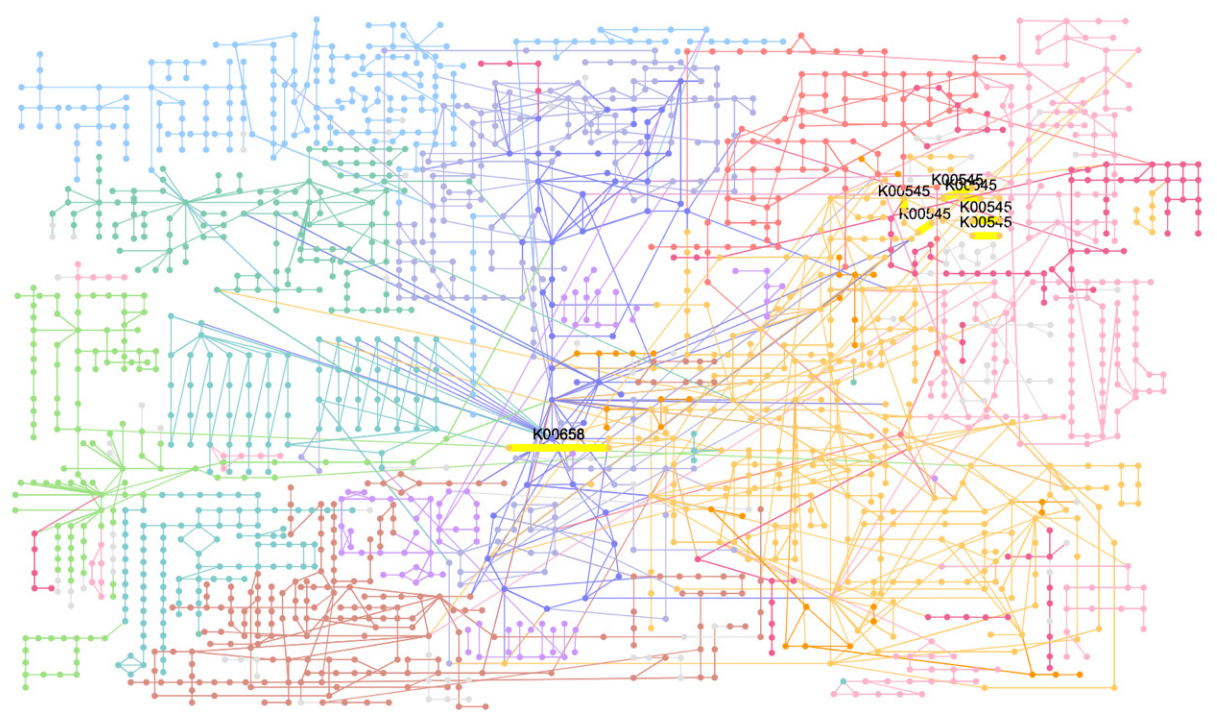

Fig. 29. Pathway for 7 chosen genes on MicrobiomeAnalyst.

Throughout the 7 genes chosen from 1.1 and 1.2, only two genes showed pathways on Microbiomeanalyst pathways which are DLST (K00658) and COMT (K00545).

Table 8. MicrobiomeAnalyst Pathways chart

\begin{tabular}{|c|c|c|}
\hline Name & Hits & P-Value \\
\hline
\end{tabular}

\begin{tabular}{|c|l|c|}
\hline $\begin{array}{c}\text { Betalain } \\
\text { biosynthesis }\end{array}$ & 1 & 0.0027 \\
\hline Lysine degradation & 1 & 0.0268 \\
\hline $\begin{array}{c}\text { Steroid hormone } \\
\text { biosymthesis }\end{array}$ & 1 & 0.0295 \\
\hline $\begin{array}{c}\text { Citrate cycle (TCA } \\
\text { cycle) }\end{array}$ & 1 & 0.0471 \\
\hline Tyrosine & 1 & 0.048 \\
\hline
\end{tabular}




\begin{tabular}{|c|c|c|}
\hline metabolism & & \\
\hline Carbon metabolism & 1 & 0.212 \\
\hline
\end{tabular}

Note. Names for the pathways found in Microbiome Analyst

There is a total of 6 pathways found in MicrobiomeAnalyst. Within these 6 pathways, there are 5 pathways that are significant. This pathway further confirms that Citric Acid Cycle is linked to the gut microbiota.

The first paragraph after a section or subsection should not be indented; subsequent paragraphs should be indented by $5 \mathrm{~mm}$.

\section{Discussion}

In this research, I found 7 genes that are likely to be involved in the brain-gut axis to influence MDD, which are: OLFM4, RAB27B, DLST, CPPED1, FKBP5, COMT and GSK3B (section A of Results). Through pathway and network analyses of these candidate genes, I further identified three potentially new mechanisms that might be involved in MDD (section B of Results). Although these mechanisms still need to be characterized with wet lab experiments, they have implicated promising directions of basic MDD research. Through GO analysis and gut microbiota analysis, I further identified the terms that are related to the 7 genes and classified the connection between gut microbiota (section $\mathrm{C}$ of Results).

After exploring a new mechanism that might connect the gut microbiota and the brain, there could be new antidepressant medicines that might have the function of increasing or decreasing the rate of Alpha-ketoglutarate, a molecule that determines the rate of the citric acid cycle. Alpha-ketoglutarate plays a very important role of maintains intestinal homeostasis, which we might infer that it might be linked to MDD by a decrease of Alphaketoglutarate [40]. However, this treatment solution still needs a verification in order to carry this antidepressant medicine out.

The limitation of this study is that I could not suggest specific gut bacterial species based on my study with solid evidence. This is due to the currently available microbiome dataset.

\section{Equations and mathematics}

In this research, I focused on a new perspective about some perspectives about MDD which is the connection between MDD and gut Microbiota. By using certain database to confirm the stability of the results.

Based on the above research, I think it is possible to conduct animal model experiments on 7 genes systematically, record the expression of the brain, digestive system, and the reaction of the animal when the gene was taken, and further identify if the gene is related with both gut microbiota and MDD.

\section{Acknowledgements}

Catherine Tian did the analysis of the data and the description for this research paper. Gregory Rose, the mentor, suggested the research topic, theoretical directions, and general guidance.

\section{References}

1. American Psychiatric Association, A.P.A. (2013). Diagnostic and statistical manual of mental disorders (5th ed.) (American Psychiatric Association).

2. The National Institute of Mental Health, T.N.I.o.M.H. (2017). Major Depression (National Institute of Mental Health, U.S. : Department of Health and Human Services).

3. Luo, Z., Li, Y., Hou, Y., Liu, X., Jiang, J., Wang, Y., Liu, X., Qiao, D., Dong, X., Li, R., et al. (2019). Gender-specific prevalence and associated factors of major depressive disorder and generalized anxiety disorder in a Chinese rural population: the Henan rural cohort study. BMC Public Health 19, 1744.

4. Wilson, S., Vaidyanathan, U., Miller, M.B., McGue, M., and Iacono, W.G. (2014). Premorbid risk factors for major depressive disorder: are they associated with early onset and recurrent course? Dev Psychopathol 26, 1477-1493.

5. India State-Level Disease Burden Initiative Mental Disorders, C. (2020). The burden of mental disorders across the states of India: the Global Burden of Disease Study 1990-2017. Lancet Psychiatry 7, 148-161.

6. Dewey, C. (2013). A stunning map of depression rates around the world.

7. Kerr, M. (2018). Major Depressive Disorder: Symptoms, Causes, and Treatment (Healthline: Healthline Media).

8. Harvard Heath Publishing, H.H.P. (2014). What are the real risks of antidepressants? (Harvard Health Publishing).

9. Neuman, F. (2018). The Problem with Antidepressant Drugs (Psychology Today: Sussex Publishers).

10. BrightQuest Treatment Centers, B.T.C. Major Depression and Bipolar Disorder. In BrightQuest Treatment Centers.

11. David, J.K., Ellen, F., and Mary, L.P. (2011). Major depressive disorder: new clinical, neurobiological, and treatment perspectives.

12. Varidaki, A., Mohammad, H., and Coffey, E.T. (2016). Molecular Mechanisms of Depression.

13. Rao, T.S., Asha, M.R., Ramesh, B.N., and Rao, K.S. (2008). Understanding nutrition, depression and mental illnesses. Indian J Psychiatry 50, 77-82.

14. Mills, J.G., Larkin, T.A., Deng, C., and Thomas, S.J. (2019). Weight gain in Major Depressive Disorder: 
Linking appetite and disordered eating to leptin and ghrelin. Psychiatry Res 279, 244-251.

15. Markus, M. (2018). Gut Microbiota: Definition, Importance, and Medical Uses. (Medical News Today: MediLexicon International).

16. Viome (2018). Anxiety? Depression? Is Your Gut Microbiome to Blame? (Viome).

17. Sampson, T.R., Debelius, J.W., Thron, T., Janssen, S., Shastri, G.G., Ilhan, Z.E., Challis, C., Schretter, C.E., Rocha, S., Gradinaru, V., et al. (2016). Gut Microbiota Regulate Motor Deficits and Neuroinflammation in a Model of Parkinson's Disease. Cell 167, 1469-1480 e1412.

18. Winter, G., Hart, R.A., Charlesworth, R.P.G., and Sharpley, C.F. (2018). Gut microbiome and depression: what we know and what we need to know. Rev Neurosci 29, 629-643.

19. Pinto-Sanchez, M.I., Hall, G.B., Ghajar, K., Nardelli, A., Bolino, C., Lau, J.T., Martin, F.P., Cominetti, O., Welsh, C., Rieder, A., et al. (2017). Probiotic Bifidobacterium longum NCC3001 Reduces Depression Scores and Alters Brain Activity: A Pilot Study in Patients With Irritable Bowel Syndrome. Gastroenterology 153, 448-459 e448.

20. Carsten, C., Susanne, M., Stefanie, S., Volker, A., Udo, D., and Judith, A. (2018). Mitochondria, Microglia, and the Immune System-How Are They Linked in Affective Disorders? (Frontiers: Frontiers).

21. D'Amelio, P., and Sassi, F. (2018). Gut Microbiota, Immune System, and Bone. Calcif Tissue Int 102, 415-425.

22. Sunkin, S.M., Ng, L., Lau, C., Dolbeare, T., Gilbert, T.L., Thompson, C.L., Hawrylycz, M., and Dang, C. (2013). Allen Brain Atlas: an integrated spatiotemporal portal for exploring the central nervous system. Nucleic Acids Res 41, D996-D1008.

23. Huang, D.W., Sherman, B.T., Tan, Q., Kir, J., Liu, D., Bryant, D., Guo, Y., Stephens, R., Baseler, M.W., Lane, H.C., et al. (2007). DAVID Bioinformatics Resources: expanded annotation database and novel algorithms to better extract biology from large gene lists. Nucleic Acids Res 35, W169-175.

24. Huang da, W., Sherman, B.T., and Lempicki, R.A. (2009). Bioinformatics enrichment tools: paths toward the comprehensive functional analysis of large gene lists. Nucleic Acids Res 37, 1-13.

25. Raudvere, U., Kolberg, L., Kuzmin, I., Arak, T., Adler, P., Peterson, H., and Vilo, J. (2019). $\mathrm{g}$ :Profiler: a web server for functional enrichment analysis and conversions of gene lists (2019 update). Nucleic Acids Res 47, W191-W198.

26. Chong, J., Liu, P., Zhou, G., and Xia, J. (2020). Using MicrobiomeAnalyst for comprehensive statistical, functional, and meta-analysis of microbiome data. nature protocals.

27. Wray, N.R., Ripke, S., Mattheisen, M., Trzaskowski, M., Byrne, E.M., Abdellaoui, A., Adams, M.J.,
Agerbo, E., Air, T.M., Andlauer, T.M.F., et al. (2018). Genome-wide association analyses identify 44 risk variants and refine the genetic architecture of major depression. Nat Genet 50, 668-681.

28. Cheung, S.G., Goldenthal, A.R., Uhlemann, A.C., Mann, J.J., Miller, J.M., and Sublette, M.E. (2019). Systematic Review of Gut Microbiota and Major Depression. Front Psychiatry 10, 34.

29. Tozzi, L., Farrell, C., Booij, L., Doolin, K., Nemoda, Z., Szyf, M., Pomares, F.B., Chiarella, J., O'Keane, V., and Frodl, T. (2018). Epigenetic Changes of FKBP5 as a Link Connecting Genetic and Environmental Risk Factors with Structural and Functional Brain Changes in Major Depression. Neuropsychopharmacology 43, 1138-1145.

30. Otsuka, Y., Kakeda, S., Sugimoto, K., Katsuki, A., Nguyen, L.H., Igata, R., Watanabe, K., Ueda, I., Kishi, T., Iwata, N., et al. (2019). COMT polymorphism regulates the hippocampal subfield volumes in first-episode, drug-naive patients with major depressive disorder. Neuropsychiatr Dis Treat $15,1537-1545$.

31. Wang, X.Y., Chen, S.H., Zhang, Y.N., and Xu, C.F. (2018). Olfactomedin-4 in digestive diseases: A mini-review. World J Gastroenterol 24, 1881-1887.

32. Anderson, N.M., Li, D., Peng, H.L., Laroche, F.J., Mansour, M.R., Gjini, E., Aioub, M., Helman, D.J., Roderick, J.E., Cheng, T., et al. (2016). The TCA cycle transferase DLST is important for MYCmediated leukemogenesis. Leukemia 30, 1365-1374.

33. Duda, P., Hajka, D., Wojcicka, O., Rakus, D., and Gizak, A. (2020). GSK3beta: A Master Player in Depressive Disorder Pathogenesis and Treatment Responsiveness. Cells 9.

34. Inkster, B., Nichols, T.E., Saemann, P.G., Auer, D.P., Holsboer, F., Muglia, P., and Matthews, P.M. (2010). Pathway-based approaches to imaging genetics association studies: Wnt signaling, GSK3beta substrates and major depression. Neuroimage 53, 908-917.

35. Zhan, T., Rindtorff, N., and Boutros, M. (2017). Wnt signaling in cancer. Oncogene 36, 1461-1473.

36. de Sousa, R.T., Streck, E.L., Forlenza, O.V., Brunoni, A.R., Zanetti, M.V., Ferreira, G.K., Diniz, B.S., Portela, L.V., Carvalho, A.F., Zarate, C.A., Jr., et al. (2015). Regulation of leukocyte tricarboxylic acid cycle in drug-naive Bipolar Disorder. Neurosci Lett 605, 65-68.

37. Margarita Rivera, Adam E. Locke, Tanguy Corre, Darina Czamara, Christiane Wolf, Ana Ching-Lopez, Yuri Milaneschi, Stefan Kloiber, Sara CohenWoods, James Rucker, K.J.A., , et al. (2018). Interaction between the FTO gene, body mass index and depression: meta-analysis of 13701 individuals. Cambridge University.

38. Saeedi, S., Israel, S., Nagy, C., and Turecki, G. (2019). The emerging role of exosomes in mental disorders. Transl Psychiatry 9, 122. 
39. Smythies, L.E., and Smythies, J.R. (2014). Exosomes in the gut. Front Immunol 5, 104.

40. Li, S., Fu, C., Zhao, Y., and He, J. (2019). Intervention with alpha-Ketoglutarate Ameliorates Colitis-Related Colorectal Carcinoma via Modulation of the Gut Microbiome. Biomed Res Int 2019, 8020785.

41. Harvard Health Publishing, H.H.P. (2010). Ask the Doctor: What Is Hypomania? (Harvard Health).

42. Johns Hopkins University School of Medicine, J.H.U.S.o.M. (2019). New research shows Parkinson's disease origins in the gut.
43. O'Toole, P.W., and Jeffery, I.B. (2015). Gut microbiota and aging. Science 350, 1214-1215.

44. Staff, M.C. (2018). Bipolar Disorder (Mayo Clinic: Mayo Foundation for Medical Education and Research).

45. The National Institute of Mental Health, T.N.I.o.M.H. Bipolar Disorder (National Institute of Mental Health, U.S: Department of Health and Human Services).

46. Wang, H.X., and Wang, Y.P. (2016). Gut Microbiota-brain Axis. Chin Med J (Engl) 129, 2373-2380. 